\title{
ADVANCED MODELING OF THE IONOSPHERE AND UPPER ATMOSPHERE
}

Dr. Boris Khattatov

Dr. Michael Murphy

Dr. Marianna Gnedin

Dr. Tim Fuller-Rowell

Dr. Valery Yudin
Brian Cruickshank

Jason Boisvert

Huthat Yalyanam

Vijay Jayaraman

Environmental Research Technologies

1320 Pearl Street, Suite 210

Boulder, Colorado 80302

15 June 2004

Final Report

APPROVED FOR PUBLIC RELEASE; DISTRIBUTION UNLIMITED

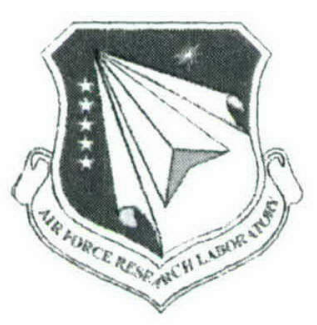

AIR FORCE RESEARCH LABORATORY Space Vehicles Directorate

29 Randolph Rd

AIR FORCE MATERIEL COMMAND

Hanscom AFB, MA 01731-3010 
This technical report has been reviewed and is approved for publication.

/Signed/

JOHN RETTERER

Conract Manager
/Signed/

ROBERT A. MORRIS

Branch Chief

This document has been reviewed by the ESC Public Affairs Office and has been approved for release to the National Technical Information Service.

Qualified requestors may obtain additional copies from the Defense Technical Information Center (DTIC). All others should apply to the National Technical Information Service.

If your address has changed, if you wish to be removed from the mailing list, or if the addressee is no longer employed by your organization, please notify AFRL/VSIM, 29 Randolph Rd., Hanscom AFB, MA 01731-3010. This will assist us in maintaining a current mailing list.

Do not return copies of this report unless contractual obligations or notices on a specific document require that it be returned. 


\section{REPORT DOCUMENTATION PAGE}

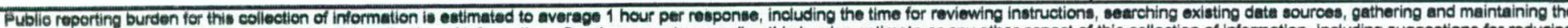

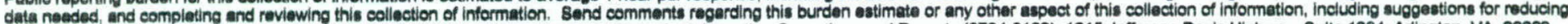

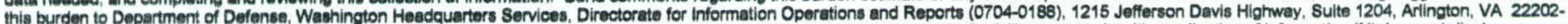

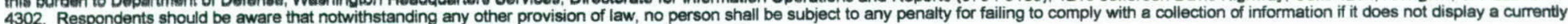
valid OMB control number. PLEASE DO NOT RETURN YOUR FORM TO THE ABOVE ADDRESS.

1. REPORT DATE (DD-MM-YYYY)

\section{REPORT TYPE}

$15-06-2004$

Final Scientific/Technical Report

3. DATES COVERED (From - TO)

15Apr2002-15Jun2004

4. TITLE AND SUBTITLE

"Advanced Modeling of the Ionosphere and Upper Atmosphere"

5a. CONTRACT NUMBER

F19628-02-C-0019

5b. GRANT NUMBER

F19628-02-C-0019

5c. PROGRAM ELEMENT NUMBER

$65502 \mathrm{~F}$

6. AUTHOR(S)

Dr. Boris Khattatov

Dr. Michael Murphy

Brian Cruikshank

5d. PROJECT NUMBER

3005

Dr. Marianna Gnedin

Jason Boisvert

Dr. Tim Fuller-Rowell

Huthas Kalyanam

5e. TASK NUMBER

SD

Dr. Valery Yudin

Vijay Jayaraman

\section{PERFORMING ORGANIZATION NAME(S) AND ADDRESS(ES)}

Environmental Research Technologies

(a DBA of Fusion Numerics, Inc.)

5f. WORK UNIT NUMBER

AC

1320 Pearl Street, Suite 210

Boulder, Colorado 80302

\section{SPONSORING / MONITORING AGENCY NAME(S) AND ADDRESS(ES)}

\section{PERFORMING ORGANIZATION REPORT} NUMBER

ERT0007z

Air Force Research Lab (AFRL)

29 Randolph Rd.

Hanscom AFB, MA

10. SPONSOR/MONITOR'S ACRONYM(S)

11. SPONSOR/MONITOR'S REPORT

NUMBER(S)

AFRL-VS-HA-TR-2004-1129

\section{DISTRIBUTION / AVAILABILITY STATEMENT}

Approved for public release; distribution unlimited.

\section{SUPPLEMENTARY NOTES}

\section{ABSTRACT}

Report developed under Small Business Innovative Research (SBIR) contract Topic AF02-038.

This report presents final results of a 2-year AFRL-sponsored project whose objective was to develop advanced modeling and data assimilation capabilities for the ionosphere and upper atmosphere. In the course of this project Fusion Numerics developed a new global threedimensional numerical model of the ionosphere, novel ionospheric data assimilation software and methodology, and an infrastructure for obtaining and managing IGS reference station data for the system.

\section{SUBJECT TERMS}

SBIR report, Ionosphere, Modeling, Electron Content, Assimilation, GPS, HF, Real-time Ionosphere Specifications

\section{Unclassified}

16. SECURITY CLASSIFICATION OF:

\begin{tabular}{l|l|}
\hline $\begin{array}{l}\text { a. REPORT } \\
\text { Unclassified }\end{array}$ & $\begin{array}{l}\text { b. ABSTRACT } \\
\text { Unclassified }\end{array}$ \\
\hline
\end{tabular}

\section{LIMITATION OF ABSTRACT}

SAR
18. NUMBER OF PAGES

43 19a. NAME OF RESPONSIBLE PERSON John Retterer 19b. TELEPHONE NUMBER (include area code)

(718) 377-3891 


\section{Contents}

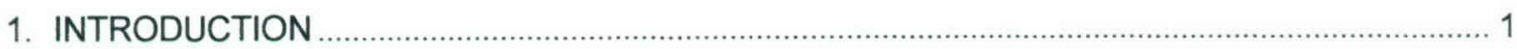

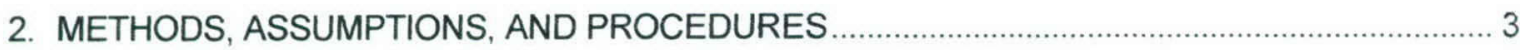

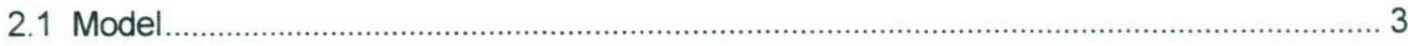

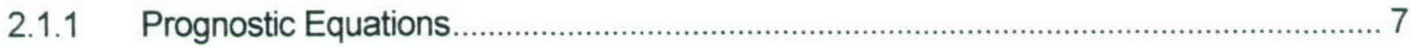

2.1.2 Fast solution of tridiagonal linear systems ...............................................................

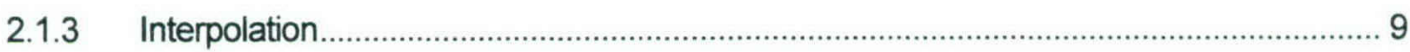

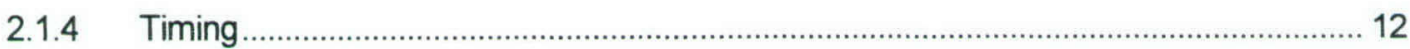

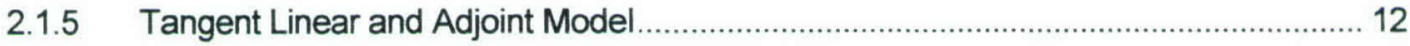

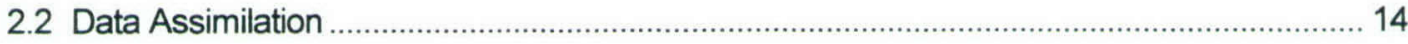

2.2.1 Kalman Filter and Sparse-Background Error Covariance ........................................... 15

2.2.2 Slant TEC determination from the IGS Global Positioning System data.................... 18

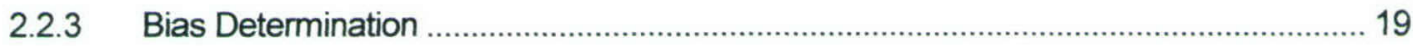

2.3 Data

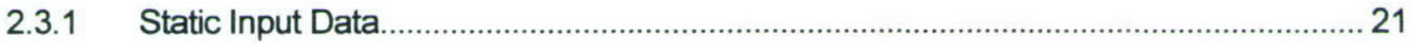

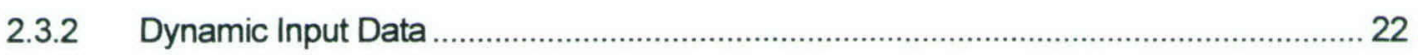

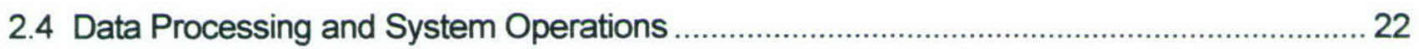

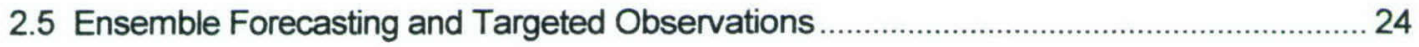

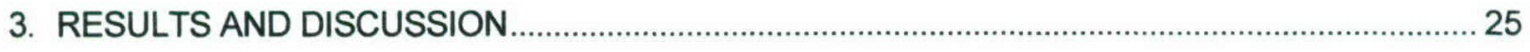

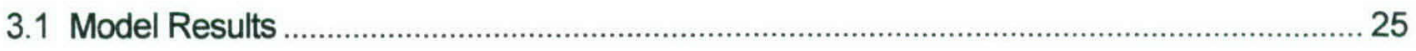

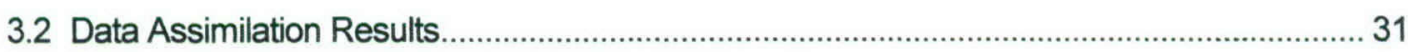

3.3 Ensemble Forecasting and Targeted Observations.......................................................... 37

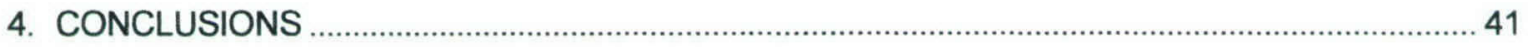

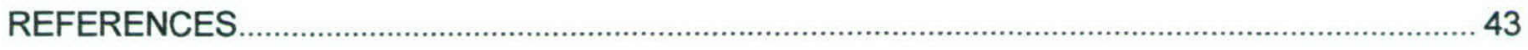




\section{Figures}

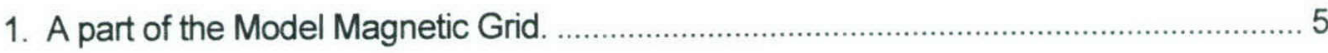

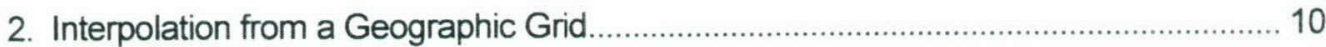

3. The Delaunay triangulation of a portion of a set of flow tubes with the same magnetic longitude.

4. An example of logarithm of absolute value of linearization matrix for one plasma tube.

5. An example of $q-p$ and magnetic longitude - $q$ cross-sections of background error covariance between a fixed point in the model grid and all other grid points.

6. Examples of bias estimation obtained in the system (black line) are shown next for two different stations together with bias estimation results from other institutions when available (JPL - magenta; CODE - green). ................................................... 20

7. Data Flow in the System. 23

8. Examples of instantaneous model prognostic variables for electrons and major ions shown at a fixed magnetic longitude.

9. Examples of instantaneous model prognostic variables for minor ions shown at a fixed magnetic longitude.

10. Examples of model-generated 3-D instantaneous iso-surfaces of electron density

11. Examples of model-generated total vertical electron content.

12. Time series of Bear Lake dynasonde measurements (red dots) and model simulated vertical TEC (solid blues).

13. Electron density profile measured by Bear Lake dynasonde (red) and simulated in the model (blue) 29

14. The $x, y$, and $z$-components of the Earth's magnetic field at the North Pole 30

15. The $x$ - and $y$-component of the Weimer electric potential at the North Pole. 30

16. Calculated $x-, y-$, and $z$ - component of the ExB drift velocities near the North Pole... 30

17. Trajectories of particles in the magnetic field acting under ExB drift with different starting locations near the North Pole.....

18. Examples of 3-D instantaneous iso-surfaces of electron density after assimilation. .. 31

19. Examples of total vertical electron content after assimilation

20. Typical time series of slant TEC from the assimilation for all satellites in view (different colors) for six different reference stations 
21. An example of time evolution of slant TEC from the assimilation system (solid lines) and GPS reference station measurements (dotted line) to several GPS satellites in view.

22. Time series of $\chi^{2}$

23. Average absolute root-mean squared (RMS) error for assimilation experiments with two different sets of de-correlation lengths

24. Average relative root-mean-squared (RMS) error for assimilation experiments with two different sets of de-correlation lengths.

25. Maximum (worst) absolute error for assimilation experiments with two different sets of de-correlation lengths.

26. Locations of IGS Stations Used in the Assimilation Process.

27. The distributions of TEC (a), and leading singular vectors of C-matrix for $20 \%$ (b), $10 \%$ (c), and $5 \%$ (d), for initial stochastic perturbations of electron density (10 members $/ 2$ hours)

28. The distributions of TEC after 6 hours of ensemble integrations

29. The longitude-altitude structure of the electron density (a) and leading singular vectors of C-matrix for $20 \%$ (b), $10 \%$ (c), and $5 \%$ (d), for initial stochastic perturbations of electron density (10 member / 2 hours).

30. The latitude-altitude structure of the electron density (a), leading singular vectors of C-matrix for $20 \%$ (b), $10 \%$ (c), and $5 \%$ (d) for initial stochastic perturbations of electron density (10 member / 2 hours)..

\section{Tables}

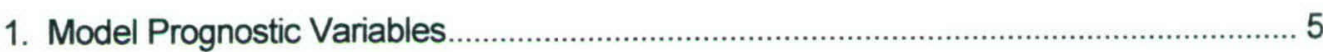

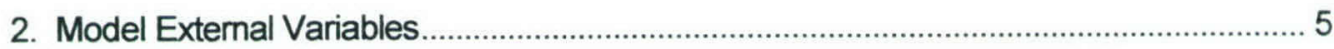

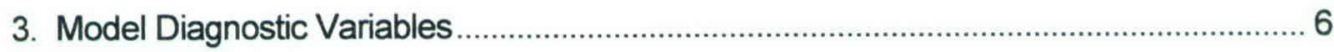

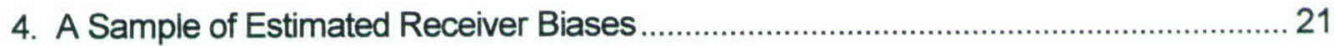




\section{INTRODUCTION}

Accurate knowledge of electron densities in the ionosphere is important for enabling fast high-accuracy positioning, reliable ground-to-satellite communications, and surveillance. Thus, an ability to forecast global and regional ionospheric conditions has applications in many areas of military operations, from $\mathrm{HF}$ communications for delivering vital information to soldiers in remote battlefields, to precisionguided weapons, space-based intelligence gathering, and space-object tracking.

The atmosphere, including the ionosphere, is a chaotic system; small errors in the initial conditions of a forecast can, under some circumstances, grow rapidly and affect predictability. Furthermore, predictability is limited by errors due to the approximate simulation of relevant physical processes in the numerical models and to poorly known external forcing. A process known as data assimilation aims to decrease these uncertainties by using observations in order to obtain better initial conditions and/or to provide better estimates of poorly known empirical quantities in parameterizations of various physical processes in the models. Fundamentals of data assimilation were developed for numerical weather prediction (NWP) and have been in use for some time. Modern NWP models include 1-10 million gridpoints and initial atmospheric states (temperature, humidity, etc) need to be specified at each of these grid-points for forecasting. It is clearly impossible to have an observational network of this size colocated with model grid-points. Additionally, meteorological data (both ground-based and satellitecollected) often have large and sometimes poorly known systematic and random errors.

Significant improvements in modern NWP have been driven, in large part, by advances in the data assimilation methodology. In very simplistic terms, data assimilation aims to: (1) use modelencapsulated knowledge of underlying atmospheric physics to interpolate between observations; (2) use Bayesian statistics to continuously combine model state with observations thus producing a less uncertain state; and (3) compute temporal evolution and spatial distribution of model errors (error covariances) to keep track of forecast quality and to be able to reject (or correct) inconsistent observations.

In order to apply this methodology to ionospheric forecasting one needs a numerical model of the ionosphere. While present meteorological forecast models are very mature, driven by the need for a reliable weather forecasting, ionospheric numerical models are mainly academic and not as advanced. Even less advanced are the specific data assimilation techniques that can be applied to the ionosphere. Additionally, in meteorology one has to solve fluid dynamics equations for only two constituents, air and water vapor. Modern ionospheric physics has to consider separately seven different ions, all free electrons, seven neutral particles, and the interactions between them which adds significant complexity.

The amount of ions and electrons in a particular region of the ionosphere is governed by a multitude of complex physical and chemical processes. Charged particles appear when Sun-emitted photons strike neutral atoms or molecules in the atmosphere and ionize them. Since the sunlight intensity increases with altitude but the amount of neutral particles decreases, electron concentrations peak at about 300 $\mathrm{km}$. Once ions and electrons have appeared, they interact with each other and neutral particles along with the Earth's magnetic field in a complex way. Additional processes need to be considered in the polar and equatorial regions. All these interactions result in fast temporal changes and often sharp horizontal gradients in the electron content; an excellent review of ionospheric processes can be found in Schunk and Nagy, (2000). Building a numerical model simulating all these processes and assuring that the resulting code is computationally efficient is a difficult task.

This report describes a global three-dimensional numerical model of the ionosphere and a data assimilation methodology developed by Fusion Numerics Inc. The model, sponsored by the US Air Force, addresses GPS receiver data assimilation and bias estimation methodologies. A part of the 
system has been operational since August 2003 and can provide real-time slant TEC from any location on Earth to all visible GPS satellites: http://fusionnumerics.com/ionosphere.

Similar research projects have been undertaken earlier as a part of the Air Force Multi-Disciplinary University Research Initiative GAIM (Global Assimilation of Ionospheric Measurements; e.g., http://genesis2.jpl.nasa.gov/archive/200212031/,

http:/www.cosis.net/abstracts/EAE03/12614/EAE03-J-12614.pdf). The current project is somewhat parallel to that effort. We addressed quite differently a number of important issues related to numerical representation of ionospheric physics in the model and implementation of data assimilation. Some of our main objectives were creating a modern, well documented, and maintainable numerical ionospheric model and its early operational implementation. Note that as of this writing ionospheric specifications for the Air Force are provided operationally by PRISM (Parameterized Real-time IonoSpheric Model). One of the objectives of the Air Force program solicitations that funded this effort was to build a prototype of the next generation operational ionospheric model capable of assimilating different types of real-time data.

Implementation of practical and efficient data assimilation schemes as well as operational implementations of forecast systems depends critically on the design, quality, and maintainability of the underlying physical propagator model. During the first year of this AFRL-sponsored investigation we developed a new computer model for simulating time evolution of ion and electron densities in the ionosphere on a global scale and developed the corresponding tangent linear and adjoint models with respect to parallel transport. The core ionospheric model solves plasma dynamics and composition equations governing evolution of density, velocity and temperature for seven ion species and electrons on a fixed grid in magnetic coordinates. It uses a realistic model of the Earth's magnetic field and solar indices obtained in real time from NOAA's Space Environment Center. At the present time the model computes real-time ion and electron densities at a grid of more than one million points. Higher resolutions are anticipated in the future. During the second year, we developed and tested a new largescale suboptimal Kalman Filter data assimilation module for systematically adjusting a model-derived state (3-D fields of electron densities) with slant total electron content (TEC) measured by a network of dual frequency geographically distributed GPS receivers.

Results of the 2-year investigation are described in this report. Some of these results have also been presented at the IEEE Position, Location and Navigation 2004 (PLANS 2004) symposium (Khattatov et al, 2004), and European Navigation Conference 2004 (ENC 2004). A published paper from the proceedings of PLANS 2004 can be found in the appendix of this report.

In the beginning of this investigation we decided that the computer code of the ionospheric model available to us, Coupled Thermosphere Ionosphere Model (CTIM), was unacceptable for the purpose of adding data assimilation capabilities and practical implementation of the forecast system for several reasons, with the major reasons listed below:

- Very poor quality of code leading to inefficiencies and redundancy, rendering the code hard to understand, maintain, and modify.

- Almost complete lack of documentation.

- Extensive use of FORTRAN 77 and FORTRAN 66 features that are being phased out in the modern and future FORTRAN compiler versions.

Thus, the first major task of this investigation was creating a new set of computer code implementing the forward model and its validation. Certain features of the model that are deemed as non-critical remain in the development stage (e.g., correct calculations of night time ionization rates) and will be added later. While development of the propagator model "from scratch" took time, we deem it to be a 
critical component that enabled and strongly facilitated reaching the final objective - building a modern and operational data assimilation and forecasting system of the ionosphere.

Traditionally, development of such models in academia took decades and the efforts of many scientists. While resulting computer codes would be cutting edge in the scientific sense, they usually would be not particularly computationally efficient, user friendly or well documented. Consequentially, it would be difficult to maintain and advance such codes or build operational systems based upon them. Our approach involved reviewing key scientific publications describing relevant ionospheric physics and capturing the described concepts and equations in a series of "use-cases." These use-cases were later coded by professional software engineers and computer scientists in a modern object-oriented fashion.

As a result of this approach, which clearly would not be possible without many years of governmentsponsored academic research, energy, and efforts of many devoted scientists around the world, Fusion Numerics has built a modern numerical ionospheric model in a little over a year and then coupled it with an efficient data assimilation scheme. Web-based access to the system is provided to early users for validation and exploration purposes at http://fusionnumerics.com/ionosphere.

\section{METHODS, ASSUMPTIONS, AND PROCEDURES}

\subsection{Model}

The developed model is a numerical global model of the ionosphere loosely based on a description given in Millward et al (1996) and Bailey and Balan et al (1996). It consists of over 60,000 lines of $\mathrm{C}++$ code and was ported to SuSe Enterprise 64 bit Linux, SuSe and RedHat 32 bit Linux as well as MS Windows platforms. Since the code is fully 64-bit compliant and adheres to standard C++ language specifications it is expected to run on all major UNIX systems.

The code has been developed using modern software engineering tools and methodologies (OO design, Rational Unified Process, unit testing, UML diagramming and reverse engineering) and profiled and optimized with industry standard profilers (Rational Rose and Intel VTune). While adherence to industry standard large-scale software development process allowed us to develop the model in a relatively short time frame, this project would not have been possible without decades of published academic research by many scientists worldwide and respective financial support from various sponsors.

The model computes spatial distribution and temporal evolution of seven major ions $(\mathrm{H}+, \mathrm{O}+\mathrm{He}+$, $\mathrm{O}_{2}+, \mathrm{NO}+, \mathrm{N}_{2}+, \mathrm{N}+$ ) and electrons. Other prognostic variables include ion and electron temperatures and velocities. The model solves plasma dynamics equations for all seven ion species and electrons and an energy conservation equation for the three major ions and electrons. It includes chemical interactions with neutrals and ions, recombination, ion-ion and ion-neutral collision rates, photoionization, and different types of heating. The model variables are described in Table 1, Table 2 and Table 3.

The model domain covers all latitudes and longitudes; however implementation of polar transport and high-latitude effects terms are still in the experimental stage and should not be relied upon. As customary in ionospheric applications, the dynamic equations are solved in so-called magnetic coordinates since in the absence of electric fields plasma predominantly moves parallel to the direction of the magnetic field. A detailed discussion of the coordinate transformation and related equations can 
be found, for instance, in Millward et al (1996) and Bailey and Balan et al (1996). Here we give only a brief overview for the benefit of readers not familiar with this subject.

The Earth's magnetic field is being approximated as that of a tilted eccentric dipole. The first magnetic coordinate is magnetic longitude. For each magnetic longitude we consider a "stack" of magnetic field lines characterized by the distance of the apex of each line from the Earth's center at the magnetic equator. This distance, normalized by the Earth's radius, is the second magnetic coordinate, $p$. Finally, for each field line the distance from the apex to a particular point along the line gives the third coordinate, $s$.

A portion of the model grid for low-latitudes only is shown in

Figure 1 . For clarity only 20 longitudes and $30 p$ values are shown.

The model solves several types of prognostic equations. These equations are given in dipole coordinates, along magnetic field lines. Therefore there is only one dependent spatial coordinate corresponding to the position along the magnetic field line, $s$.

Once the field-aligned transport is solved, we compute plasma evolution due to cross-field transport. Cross-field transport is forced by electric fields either imposed externally from the magnetosphere or generated internally from the action of the neutral wind. In the lower thermosphere the mobility of the ions is inhibited by collisions with the neutral atmosphere. The dynamo action of the neutral winds drives currents that through continuity create polarization electric fields. The ions respond to these electric fields by drifting perpendicular to both the electric and magnetic fields.

This is often referred to as ExB transport. In non-fully coupled models the ExB plasma velocity is specified from external empirical models. Once this velocity is known, solving plasma advection equation is relatively straightforward. To model ExB drif at low latitudes we use coefficients of the Fejer and Schierless model. To model ExB drift in the polar regions we use coefficients of the Weimer model provided by Dr. Dan Weimer of Mission Research Inc.

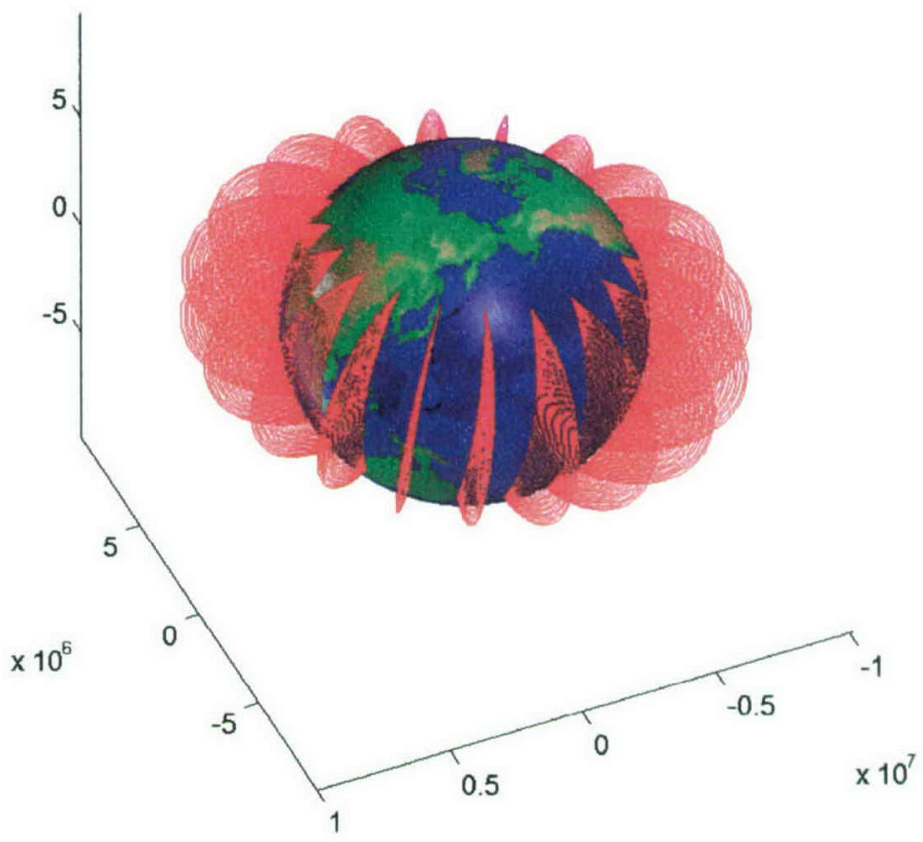

Figure 1. A Part of the Model Magnetic Grid 
Table 1: Model Prognostic Variables

\begin{tabular}{|c|c|c|c|}
\hline $\begin{array}{l}\text { Variable } \\
\text { Name }\end{array}$ & Units & Description & Comments \\
\hline Ion densities & $\begin{array}{l}\text { particles' } \\
\mathrm{m}^{3}\end{array}$ & $\begin{array}{l}\text { Local (point) volume density of a particular } \\
\text { ion species }\end{array}$ & $\begin{array}{l}\text { at present there are } 7 \\
\text { ions: } \mathrm{O}^{+} \cdot \mathrm{H}^{+}, \mathrm{He}^{+} \cdot \mathrm{N}_{2}^{+} \\
\mathrm{O}_{2}^{+}, \mathrm{NO}^{+}, \mathrm{N}^{+}\end{array}$ \\
\hline $\begin{array}{l}\text { Ion } \\
\text { temperatures }\end{array}$ & $\mathrm{K}$, degree & $\begin{array}{l}\text { Local temperature of a particular ion } \\
\text { species }\end{array}$ & same \\
\hline Ion velocities & $\mathrm{m} / \mathrm{s}$ & $\begin{array}{l}\text { Local (point) velocity of a particular ion } \\
\text { species along the magnetic field line } \\
\text { passing through this point }\end{array}$ & same \\
\hline $\begin{array}{l}\text { Electron } \\
\text { temperature }\end{array}$ & $\mathrm{K}$, degree & Local electron temperature & \\
\hline $\begin{array}{l}\text { Electron } \\
\text { velocity }\end{array}$ & $\mathrm{m} / \mathrm{s}$ & $\begin{array}{l}\text { Local (point) velocity of electrons along the } \\
\text { magnetic field line passing through this } \\
\text { point }\end{array}$ & \\
\hline $\begin{array}{l}\text { Electron } \\
\text { density }\end{array}$ & $\begin{array}{l}\text { particles/ } \\
\mathrm{m}^{3}\end{array}$ & Local (point) volume density of electrons & $\begin{array}{l}\text { is the sum of all local } \\
\text { ion densities }\end{array}$ \\
\hline
\end{tabular}

Table 2: Model External Variables

\begin{tabular}{|c|c|c|c|}
\hline $\begin{array}{l}\text { Variable } \\
\text { Name }\end{array}$ & Units & Description & Comments \\
\hline $\begin{array}{l}\text { Neutral } \\
\text { densities }\end{array}$ & $\begin{array}{l}\text { particles/ } \\
\mathrm{m}^{3}\end{array}$ & $\begin{array}{l}\text { Local (point) volume density } \\
\text { of a particular neutral species }\end{array}$ & $\begin{array}{l}\text { - at present there are } 7 \text { neutrals: } \\
\mathrm{O}, \mathrm{O}_{2}, \mathrm{~N}_{2}, \mathrm{He}, \mathrm{H}, \mathrm{NO}, \mathrm{N} \text {. }\end{array}$ \\
\hline $\begin{array}{l}\text { Neutral } \\
\text { temperature }\end{array}$ & $\begin{array}{l}\mathrm{K} \text {, } \\
\text { degree }\end{array}$ & $\begin{array}{l}\text { Local temperature of all } \\
\text { neutral species (one for all) }\end{array}$ & same \\
\hline $\begin{array}{l}\text { Neutral zonal } \\
\text { velocity }\end{array}$ & $\mathrm{m} / \mathrm{s}$ & $\begin{array}{l}\text { Local (point) velocity of all } \\
\text { neutral species (one for all) } \\
\text { in the zonal direction (east- } \\
\text { west, eastward is positive) }\end{array}$ & same \\
\hline $\begin{array}{l}\text { Neutral } \\
\text { meridional } \\
\text { velocity }\end{array}$ & $\mathrm{m} / \mathrm{s}$ & $\begin{array}{l}\text { Local (point) velocity of all } \\
\text { neutral species (one for all) } \\
\text { in the meridional direction } \\
\text { (north-south, northward is } \\
\text { positive) }\end{array}$ & same \\
\hline
\end{tabular}




\section{Variable Name \\ Units \\ ExB zonal velocity $\mathrm{m} / \mathrm{s}$}

ExB meridional velocity

Photo production

\section{Chemical \\ production}

Chemical loss

Photoionization
rates
Chemical reaction
rates
Recombination
reaction rates

lon-neutral

collision

frequencies

Ion-ion callision

frequencies

Ion heating rates

$\mathrm{J} / \mathrm{m}^{3} / \mathrm{s}$

lon thermal conductivities

Electron heating rates

Electron therma $\mathrm{J} / \mathrm{K} / \mathrm{m} / \mathrm{s}$

Particles $/ \mathrm{s} / \mathrm{m}^{3}$

$1 / \mathrm{s}$

$\mathrm{m}^{3} / \mathrm{s}$

$1 / \mathrm{s}$

$1 / \mathrm{s}$

$1 / \mathrm{s}$

Particles $/ \mathrm{s} / \mathrm{m}^{3}$

s

政

$\mathrm{J} / \mathrm{K} / \mathrm{m} / \mathrm{s}$

$\mathrm{J} / \mathrm{m}^{3} / \mathrm{s}$

conductivities

\section{Description}

Local (point) velocity associated with the zonal ExB drift of the magnetic field line passing through this point

Local (point) velocity associated with the meridional ExB drift of the magnetic field lime passing through this point

Number of particles of a particular ion species produced as a result of photoionization per second per unit volume.

Number of particles of a particular ion species produced as a result of chemical reactions per second per unit volume

Number of particles of a particular ion species lost as a result of chemical and recombination reactions per second per unit volume

Coefficients needed to compute photo production

Coefficients needed to compute chemical loss due to electron exchange reactions.

Coefficients needed to compute loss due to recombination chemical reactions

Drag on a particular ion particle due to collisions with a neutral species.

Drag on a particular ion particle due to collisions with a different ion species.

Heating due to Joule heating. frictional collisions and other processes.

Heating due to Joule heating, frictional collisions and other processes.

\section{Comments}

Needs to be computed from empirical ExB models

same as above

- at present there are 7 neutrals, only 5 of those can be photoionized $\mathrm{O} . \mathrm{O}_{2}, \mathrm{~N}_{2}, \mathrm{He}, \mathrm{N}$. Other ions are produced via chemical reactions, such as

$\mathrm{O}^{+}+\mathrm{H} \rightarrow \mathrm{H}^{+}+\mathrm{O}$.

There are 21 chemical reactions at the present e.g., $\mathrm{O}^{+}+\mathrm{H} \rightarrow \mathrm{H}^{+}+\mathrm{O}$.

This value is a product of the density (concentration) of the ion species being destructed and the chemical loss rate. $\boldsymbol{L}$.

- at present there are 7 neutrals. only 5 of those can be photo-ionized: $\mathrm{O}, \mathrm{O}_{2}, \mathrm{~N}_{2}, \mathrm{He}, \mathrm{N}$

There are 21 chemical reactions at the present. e.g., $\mathrm{O}^{+}+\mathrm{H} \rightarrow \mathrm{H}^{+}+\mathrm{O}$.

There are 7 recombination reactions, e.g. $\mathrm{O}^{+}+$ $e \rightarrow 0$.

e represents an electron.

There are 7 ions and 7 neutrals, therefore it is a $7 \times 7$ matrix with zero diagonal.

There are 7 ions, therefore it is a $7 \times 7$ matrix with zero diagonal.

Is only computed for three major ions, $\mathrm{O}^{+}, \mathrm{H}^{+}$, $\mathrm{He}^{+}$

Is only computed for three major ions, $\mathrm{O}^{+}, \mathrm{H}^{+}$, $\mathrm{He}^{+}$ 


\subsubsection{Prognostic Equations}

A prognostic equation allows one to estimate a particular prognostic variable at a future time. The prognostic variables are density, velocity and temperature for ions and electron density, temperature and velocity.

These equations are given in dipole coordinates, along magnetic flow tubes. Therefore, there is only one dependent spatial coordinate corresponding to the position along the magnetic flow tube. This can be a non-dimensional variable $\mathrm{q}$ or a dimensional variable $s=q \cdot \mathrm{R}_{\mathrm{e}}\left(\mathrm{R}_{\mathrm{e}}\right.$ is the radius of the earth),

\subsubsection{Continuity Equation For Each Ion Species}

Numerical solution of this equation should generate ion density $N_{i}(t+\square t)$ given all related variables at time $t$.

$$
\frac{\partial N_{i}}{\partial t}-b_{s}^{2} \frac{\partial\left(\frac{N_{i} V_{i}}{b_{s}}\right)}{\partial s}+N_{i} \cdot \nabla V_{\perp}+\nabla N_{i} \cdot V_{\perp}=P_{i}-L_{i} \cdot N_{i}
$$

where

$$
\begin{aligned}
& N_{i}-\text { density of ion } \mathrm{i} \\
& V_{i}-\text { velocity (aligned with the magnetic flow tube) of ion } \mathrm{i} \\
& s=q \cdot \mathrm{R}_{\mathrm{e}} \\
& b_{s}=\sqrt{1+3 \cos ^{2}(\text { eccLat })} \cdot\left(\frac{\mathrm{R}_{\mathrm{e}}}{\text { eccRadius }}\right)^{3} \\
& P_{i} \text { - chemical production + photochemical production } \\
& L_{i}-\text { chemical loss rate } \\
& L_{i} \cdot N_{i} \text { - chemical loss }
\end{aligned}
$$

The term

$$
\nabla V_{\perp}=\frac{6 \cdot V_{\perp}^{e q} \sin ^{2}(e c c L a t) \cdot\left(1+\cos ^{2}(e c c L a t)\right)}{p \cdot R_{e} \cdot\left(1+3 \cdot \cos ^{2}(e c c L a t)\right)^{2}}
$$

is a divergence of $\mathrm{ExB}$ velocity in the vertical (and meridional) plane, i.e., in p direction. $V_{\perp}^{e q}$ is the value of ExB meridional drift at the magnetic equator corresponding to a particular $\mathrm{p}$.

\subsubsection{Momentum Equation For Each Ion Species}

Numerical solution of this equation should generate ion velocity $V_{i}(t+\square t)$ given all related variables at time $t$. 


$$
V_{i}=\frac{1}{\sum_{n=1}^{N_{-} \text {Neutrals }} v_{i n}+\sum_{j=1}^{N} \sum_{i j}^{\text {lons }} v_{i j}} \cdot\left[\begin{array}{l}
-g \sin I+\frac{b_{s} k_{i}}{m_{i}}\left(\frac{T_{i}}{N_{i}} \frac{\partial N_{i}}{\partial s}+\frac{T_{e}}{N_{e}} \frac{\partial N_{e}}{\partial s}+\frac{\partial\left(T_{i}+T_{e}\right)}{\partial s}\right) \\
+\sum_{n=1}^{N_{-}} v_{i n}^{\text {Neurrals }} v_{n}\left(V_{n} \cos D-U_{n} \sin D\right) \cos I+\sum_{j=1}^{N} v_{i j}^{\text {lons }} V_{j}
\end{array}\right]
$$

where

$$
\begin{aligned}
& N_{i}-\text { density of ion } \mathrm{i} \\
& V_{i}-\text { velocity (aligned with the magnetic flow tube) of ion } \mathrm{i} \\
& V_{j}-\text { velocity of ion } \mathrm{j} . \\
& s=q \cdot \mathrm{R}_{\mathrm{e}} \\
& b_{s}=\sqrt{1+3 \cos ^{2}(e c c L a t)} \cdot\left(\frac{\mathrm{R}_{\mathrm{e}}}{e c c \text { Radius }}\right)^{3} \\
& m_{i}-\text { mass of ion i. } \\
& \mathrm{k}-\text { Boltzmann's constant. } \\
& T_{i}-\text { temperature of ion i. } \\
& T_{e}-\text { electron temperature } \\
& N_{e}-\text { electron density. } \\
& U_{n}-\text { zonal neutral velocity. } \\
& V_{n}-\text { meridional neutral velocity. } \\
& v_{i n}-\text { ion-neutral collision frequency. } \\
& v_{i j} \text { - ion-ion collision frequency. } \\
& \mathrm{g}-\text { acceleration of gravity. } \\
& \mathrm{I}-\text { inclination angle for this flow tube: } \\
& \sin I=\frac{2 \cos (e c c L a t)}{\sqrt{1+3 \cos ^{2}(e c c L a t)}} \\
& \cos I=\frac{\sin (e c c L a t)}{\sqrt{1+3 \cos ^{2}(e c c L a t)}} \\
& \mathrm{D}-\text { declination angle for this flow tube. }
\end{aligned}
$$

\subsubsection{Energy Equation for Each Ion Species}

Numerical solution of this equation generates ion temperature $T_{i}(t+\square t)$ given all related variables at time $t$. The temperature equation is solved for three major ions $\left(\mathrm{O}^{+}, \mathrm{H}^{+}\right.$and $\left.\mathrm{He}^{+}\right)$. Temperatures of the remaining ions are set to temperature of $\mathrm{O}^{+}$.

$$
\frac{3}{2} k N_{i}\left(\frac{\partial T_{i}}{\partial t}+V_{\perp} \nabla T_{i}\right)=k N_{i} T_{i} b_{s}^{2} \frac{\partial}{\partial s}\left(\frac{V_{i}}{b_{s}}\right)-k N_{i} T_{i} \cdot \nabla V_{\perp}+b_{s}^{2} \frac{\partial}{\partial s}\left(\kappa \frac{\partial T_{i}}{\partial s}\right)+\frac{3}{2} k N_{i} V_{i} b_{s} \frac{\partial T_{i}}{\partial s}+Q+F
$$


Where

$\mathrm{Q}$ and $\mathrm{F}$ - are different types of heating rates

$K_{-}$- is the thermal conductivity

\subsection{Electron Temperature Equation}

It is similar to ion temperature equation, except that the conductivities and heating rates are computed for electrons.

\subsection{Electron Density Equation}

$$
N_{e}=\sum_{i=1}^{\text {NumberOffons }} N_{i}
$$

\subsection{Electron Velocity Equation}

Assumes that there are no field-aligned currents:

$$
V_{e}=\frac{\sum_{i=1}^{\text {NumberOflons }} V_{i} N_{i}}{N_{e}}
$$

\subsubsection{Fast solution of tridiagonal linear systems}

After discretization of the prognostic equations we obtain a system of linear equations which has the tridiagonal structure. A square matrix $A=\left(a_{i j}\right)$ is said to be tridiagonal if $a_{i j}=0$ for all pairs $(i, j)$ satisfying $|i-j|>0$. Thus in the $i$ th row, only $a_{i, i-1}$ and $a_{i i}$ and $a_{i, i+l}$ can be different from zero. Three vectors can be used to store the nonzero elements.

Using standard sweep solver, such systems can be solved very quickly. In fact, the solution only requires linear time, as opposed to cubic time, in the general case. However, if the matrix is illconditioned, then the solution can be inaccurate. To remedy this, we explored the sophisticated pivoting algorithms of LAPACK, which implement careful pivoting and are robust against illconditioned matrices but require longer execution time. We determined that simple sweep solver was sufficient. The code also implements error monitoring.

\subsubsection{Interpolation}

The problem of interpolating a value at an unknown point in the ionosphere arises in two important tasks in the system. The first problem is to interpolate information about the neutral atmosphere, stored in a regular geographic grid onto each point in the magnetic global atmosphere grid. This problem is relatively straightforward because of the regular structure of the grid points.

A major task involves interpolating values from a magnetic (dipole) grid. This is required for presenting model results on a geographic grid. Also, when computing slant TEC, one needs the value along an almost arbitrary line segment passing through the ionosphere for integration. This is needed 
for assimilating TEC readings from ground stations. This problem is more difficult because there is little structure of the density of the points to exploit. For this we needed more sophisticated geometric algorithms.

We describe both of these solutions below.

\subsubsection{Spherical Interpolation}

We use "Spherical Interpolation" to interpolate a value from a regular geographic grid. Because this grid locally has the structure of a Cartesian grid, it is straightforward to locate the cube containing the query point. The interpolated function values at the query point can be obtained by using trilinear interpolation.

That is, suppose we have a point at position $(x, y, z)$ located inside the cube in Figure 2.

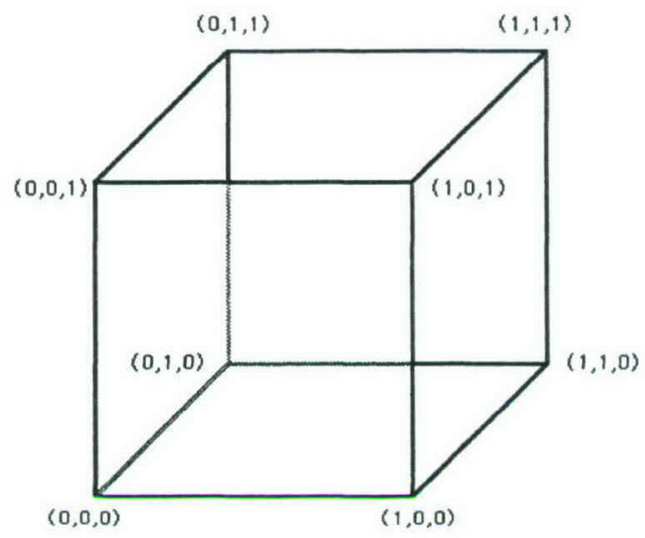

Figure 2: Interpolation from a Geographic Grid.

The value of the function we are interpolating at position $(x, y, z)$ within the cube will be denoted $V_{x y z}$ and is given by :

$$
\begin{aligned}
\mathrm{V}_{\mathrm{xyz}}= & V_{000}(1-\mathrm{x})(1-\mathrm{y})(1-\mathrm{z})+ \\
& \mathrm{V}_{100} \mathrm{x}(1-\mathrm{y})(1-\mathrm{z})+ \\
& \mathrm{V}_{010}(1-\mathrm{x}) \mathrm{y}(1-\mathrm{z})+ \\
& \mathrm{V}_{001}(1-\mathrm{x})(1-\mathrm{y}) \mathrm{z}+ \\
& \mathrm{V}_{101} \mathrm{x}(1-\mathrm{y}) \mathrm{z}+ \\
& \mathrm{V}_{011}(1-\mathrm{x}) \mathrm{yz}+ \\
& \mathrm{V}_{110} \mathrm{xy}(1-\mathrm{z})+ \\
& V_{111} \mathrm{xyz}
\end{aligned}
$$

Although technically, the "cubes" in the geographic grid are not really cubes due to the earth's curvature, the density of the grid allowed us to treat them as cubes with negligible error. Indeed, our algorithm compared well with the interpolation operations in MATLAB. 


\subsubsection{Interpolation with scattered data}

When interpolating from a magnetic grid, the problem is much more complicated, because there is not much regularity in the point spacing.

How do we interpolate a function when we know the function at points whose location has very little structure to exploit? We make use of a fundamental geometric concept known as a triangulation of a set of points. A set of triangles meeting at edges and vertices whose union equals the domain is a working definition of a triangulation. When we speak of triangulations of point sets, we mean that we wish to form triangulation so that the points are the vertices of the triangles, with the boundary edges being the edges on the convex hull of the points. (The convex hull is the smallest convex set containing the point set.)

Having a triangulation of a set of points in the plane, allows us to locate the triangle containing the query point, and then writing the query point in terms of the barycentric coordinates of that triangle. We can then use the barycentric coordinates to weight the contribution of the function values for those three points. This is piece-wise linear interpolation.

Because a set of points in the plane can be triangulated many ways, what is the best triangulation? This problem has been considered for many years and it is commonly accepted that the Delaunay triangulation has many nice properties for interpolation. Further, it is very easy to compute. In our code, we used the implementation given in Graphics Gems I. Figure 3 shows the Delaunay triangulation of a set of flow tubes of nearly equal longitude, projected into the plane.

While two-dimensional Delaunay triangulations are well-suited for interpolating two-dimensional functions, the three-dimensional Delaunay tetrahedralization, is not as easy to work with. The biggest problem is that they are more difficult to compute. Indeed, they would consume the bulk of processing time if we had put them into the model. To retain the desirable interpolation properties, we exploited the fact that a set of flow tubes share constant magnetic longitude. So to interpolate a given point, we found the two slices of flow tubes surrounding the query point. We then projected the query point onto these two flow tubes, performed barycentric interpolation, and then weighted the two interpolations based on the inverse distance from the query point to the interpolated points in both sets of flow tubes. Thus, rather than using four interpolating points in the magnetic global atmosphere we use two sets of three points on each slice. We found, that by interpolating in this way, we were nearly within agreement to the true Delaunay interpolation, and were able to process queries much faster. 


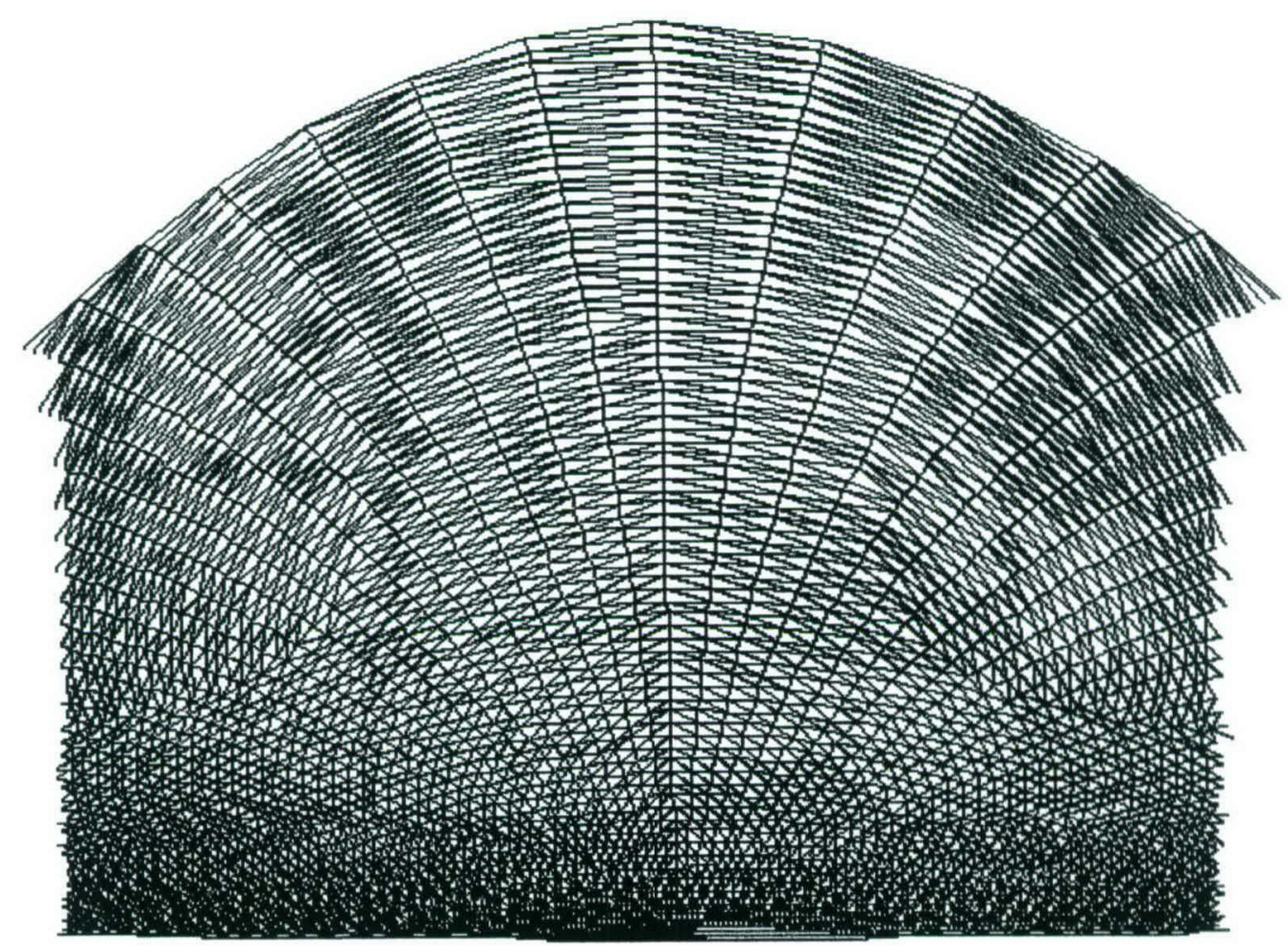

Figure 3: The Delaunay triangulation of a portion of a set of flow tubes with the same magnetic longitude.

\subsubsection{Line-of-Sight Calculations}

To compute the slant TEC from the model, we had to identify the coordinates of the ground-station and the satellite position. We then discretized the line segment connecting these two points, and interpolated the electron density from the model magnetic grid to each one of these points along the segment. Since the same magnetic grid point can contribute to interpolated values at several segments, we then consolidate all weights and store only one weight per grid point. These weights together with indices of the corresponding points constitute observational operator in the Kalman filter section of the system.

From there, we used the composite-trapezoid rule to integrate the density from each of these interpolated values and obtain a model-simulated slant TEC. We explored a variety of step sizes when discretizing the line segment, and chose the optimal tradeoff between accuracy and running time.

\subsubsection{Timing}

On a Pentium Xeon 2.2GHz computer, 1 model time step requires 35 seconds in a configuration with $10^{6}$ grid points and the memory footprint is $1.5 \mathrm{~Gb}$.

\subsubsection{Tangent Linear and Adjoint Model}

We consider two approaches to computing evolution of background error covariance in the system. The first is described in Khattatov et al (2000), where a modified tracer mass conservation equation is used to model the variance. Off-diagonals of the error covariance matrix are then obtained from values of the 
variance using an assumption of Gaussian de-correlation. The second approach, described in this section, involves obtaining the tangent-linear of the underlying model, either explicitly as a sparse matrix, or implicitly in the form of a code that computes a product of the linearization matrix and an arbitrary vector. After such linearization matrix is obtained time evolution of the error covariance can be explicitly computed as described, for instance, in Khattatov et al (2000). After some experimentation we decided that the first approach is more practical, while the second should be used for exploring and tuning the optimal values of the de-correlation lengths. We therefore describe calculations of the linearization matrix with respect to the mass-conservation equation for a particular plasma flow tube.

Let us assume that the model $\mathbf{M}$ is a forward time-dependent discrete propagator that accepts the current state $\mathbf{x}_{\mathbf{t}}$ (electron or ion densities throughout the model domain arranged in a vector) and values of several atmospheric drivers $\mathbf{p}_{\mathbf{t}}$ (e.g., level of solar activity, etc) as inputs and generates state estimates for a later time:

$$
\mathbf{x}_{t+\Delta t}=\mathbf{M}\left(\mathbf{p}_{t}, \mathbf{x}_{t}\right)
$$

Both quantities, $\mathbf{x}_{\mathbf{t}}$ and $\mathbf{p}_{\mathrm{t}}$, are considered to be model parameters.

Generally speaking, the tangent linear of the model $\mathrm{M}$ is simply a derivative of the results with respect to the initial conditions or input parameters. Note that $\mathbf{M}$ is a non-linear vector function and therefore its linearizations (first derivatives) are matrices:

$$
\mathbf{L}_{\mathbf{x}}=\frac{\partial \mathbf{M}}{\partial \mathbf{x}} \text { and } \mathbf{L}_{\mathbf{p}}=\frac{\partial \mathbf{M}}{\partial \mathbf{p}}
$$

The adjoint of the propagator model is simply a transposed of the matrix L or, a way to compute a product of the transposed and an arbitrary vector. The linearization matrix describes the sensitivity of the model with respect to the initial conditions and the adjoint is used to either minimize the cost function in the variational assimilation approach or to solve the Kalman filter equations.

These linearization matrices can be obtained in several ways:

- approximated via finite-differences calculations, i.e., by introducing small changes in $\mathbf{x}_{\mathbf{t}}$ and/or $\mathbf{p}_{\mathbf{t}}$ and computing resulting changes in $\mathbf{x}_{\mathbf{t}+\Delta t}$ :

$$
\mathbf{L}_{\mathbf{x}} \approx \frac{\Delta \mathbf{M}}{\Delta \mathbf{x}_{t}}=\frac{\Delta \mathbf{x}_{t+\Delta t}}{\Delta \mathbf{x}_{t}}
$$

- by differentiating the actual computer code (e.g., Fortran or C) implementing the model and generating computer code for direct computation of $\mathbf{L}$.

- by analytical differentiation of theoretical equations of the model and coding the results.

The first approach can be extremely CPU intensive but is straightforward to implement. The other two are much more efficient but can be hard to implement and will have to be re-done if changes are introduced into the model.

We followed the second approach and obtained and coded explicit calculations of both these matrices for each plasma tube in the model. An example of the linearization matrix is shown in Figure 4 for $\mathrm{O}^{+}$. 


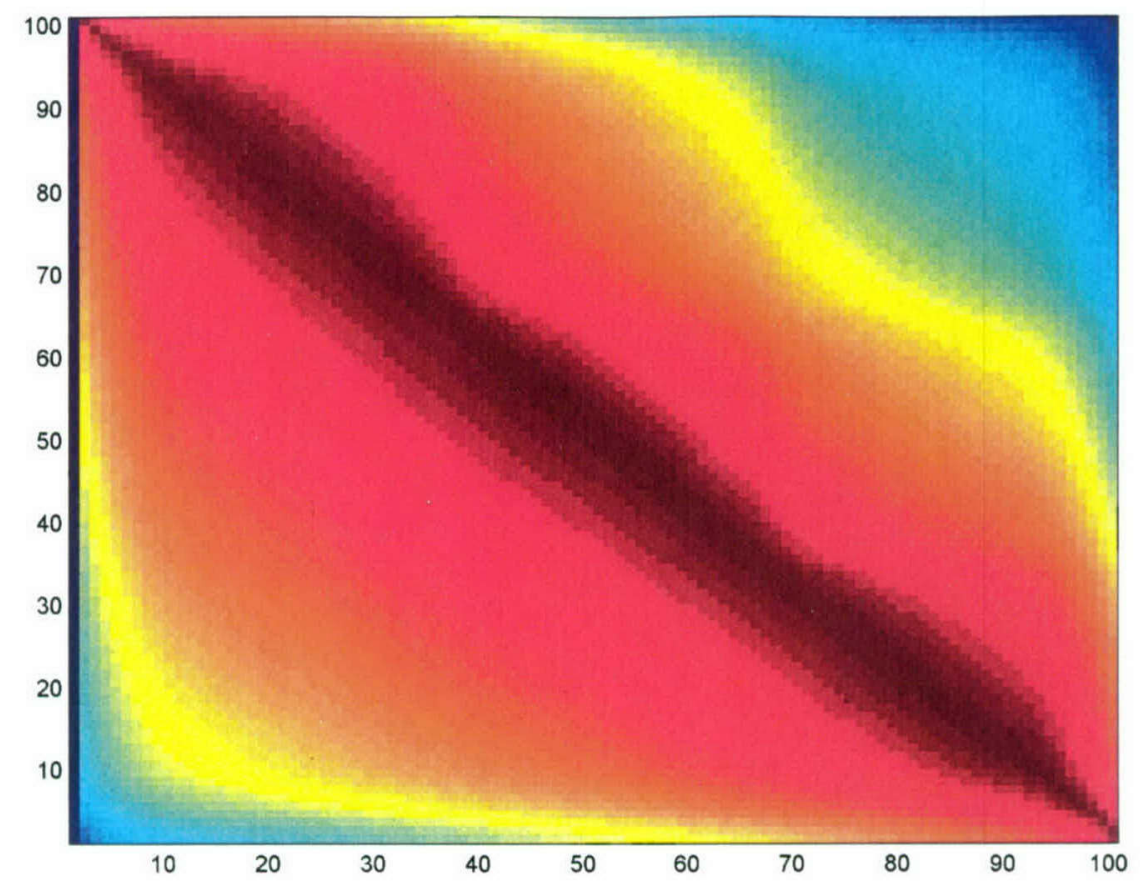

Figure 4: An example of logarithm of absolute value of linearization matrix for one plasma tube.

The particular plasma tube has 100 points. Changes in ion densities at the next time step can be obtained by multiplying this matrix by a vector of changes in ion density for this tube at a previous tie step. The asymmetries in the matrix reflect the fact that in this particular case the prevailing parallel transport is directed from the top-left end of the tube to the bottom-right.

Once the linearization matrix is obtained, evolution of the error-covariance matrices for different points on the same tube can be trivially computed as follows:

$$
\mathbf{C}(t+\Delta t)=\mathbf{L}_{\mathbf{x}} \mathbf{C}(t) \mathbf{L}_{\mathbf{x}}^{T}
$$

\subsection{Data Assimilation}

The Kalman filter is a mathematical tool traditionally used to continuously combine model predictions of the system state with noisy observations in order to come up with a less uncertain estimation of the system state and error covariances. The Kalman filter approach adopted here resembles that of Khattatov et al (2000). Let us assume that model estimates of electron densities at all grid points at time $t$ are arranged in a vector $\mathbf{x}$ with dimension $N_{x}$. Formally, integration of the model $\mathbf{M}$ can be written as

$$
\mathbf{x}_{t+\Delta t}=\mathbf{M}\left(t, \mathbf{x}_{t}\right) .
$$


Let vector $\mathbf{y}$ contain observations of a quantity linearly related to electron densities at the same time. In the case of GPS reference station data such quantities are slant TEC from each station to all satellites in view.

The connection between $\mathbf{x}$ and $\mathbf{y}$ is established via linear interpolation and integration observational operator $\mathrm{H}$ as follows:

$$
\mathbf{y}=\mathbf{H}(\mathbf{x})
$$

Under assumptions of linearity and Gaussian statistics, the optimal value of $\mathbf{x}$ that inverts (11) given a set of observations $\mathrm{y}$ and model estimates of $\mathrm{x}$ is given by the Kalman filter [9]:

$$
\begin{aligned}
& \mathbf{x}_{t}^{a}=\mathbf{x}_{t}+\mathbf{K}\left(\mathbf{y}-\mathbf{H} \mathbf{x}_{t}\right) \\
& \mathbf{K}=\mathbf{B}_{t} \mathbf{H}^{\mathrm{T}}\left(\mathbf{H} \mathbf{B}_{t} \mathbf{H}^{\mathrm{T}}+\mathbf{O}+\mathbf{R}\right)^{-1} .
\end{aligned}
$$

Here $\mathbf{B}_{t}$ is the forecast error covariance at time $t . \mathbf{O}$ is the error covariance matrix of the observations and $\mathbf{R}$ is the representativeness error covariance associated with errors of interpolation and discretization. Matrix $\mathbf{K}$ is called the Kalman gain matrix.

The analysis error covariance is expressed as:

$$
\mathbf{B}_{t}^{a}=\mathbf{B}_{t}-\mathbf{B}_{t} \mathbf{H}^{\mathrm{T}}\left(\mathbf{H B}_{t} \mathbf{H}^{\mathrm{T}}+\mathbf{O}+\mathbf{R}\right)^{-1} \mathbf{H B}_{t} .
$$

Once inversion of (11) is performed, the obtained electron densities, $\mathbf{x}_{t}^{a}$, can be used as the initial condition for the model $\mathbf{M}$ to predict electron densities at a later time (beginning of the next assimilation window) according to:

$$
\mathbf{x}_{t+\Delta t}=\mathbf{M}\left(t, \mathbf{x}_{t}^{a}\right)
$$

Since the model domain contains about $10^{6}$ points, direct matrix manipulations described by (12), (13), and (14) are impossible to implement even with modern computing capabilities. As is customary in large-scale Kalman filter implementations in NWP and other areas of atmospheric sciences, one tracks only the evolution of the diagonal of the background error covariance matrix and parameterizes the offdiagonal elements. If we also assume that correlations between variations of electron densities at two different points become negligible at certain distances (i.e., assuming compactly supported error covariance models), the matrices become sparse and the abovementioned calculations become possible.

We argue that since plasma equations are solved in magnetic coordinates, in order for the background error covariance to be separable, the de-correlation lengths and distances between points need to be specified in magnetic rather than geographic coordinates as in Hajj et al (2003). This is the approach adopted here.

\subsubsection{Kalman Filter and Sparse-Background Error Covariance}

The state vector X consists of the values of electron density $N_{e}$ in every node of the computational grid. Given the size of the state vector X (about a million entries), the model covariance matrix B (up to million by million entries), and the observational matrix $\mathrm{H}$ (up to 600 by a million entries), the exact implementation of the abovementioned equations is out of the question. 
The model covariance matrix B contains the information about the mutual correlation of the values of electronic densities in every grid cell with each other. Consequently, its size is equal to the number of grid points squared. Given the large size of this matrix, it is not feasible to properly update all of its offdiagonal elements during every Kalman Filter step. Instead, only the evolution of the variance (i.e. diagonal of the covariance matrix B) is tracked during the computation, and the off-diagonal elements are parameterized by the diagonal elements of $\mathrm{B}$ and are simply rescaled every time according to the recomputed variance. The time update is also performed for the variance only.

The off-diagonal elements $B_{i, j}$ correspond to the correlations between the corresponding nodes of the magnetic grid. Let $i$ and $j$ correspond to the two nodes of the magnetic grid with the magnetic coordinates $\operatorname{lon}_{i}, p_{i}, q_{i}$ and $l o n_{j}, p_{j}, q_{j}$, respectively. The correlation $\operatorname{Cor}_{i, j}$ between these two nodes is determined as follows:

$$
\operatorname{Cor}_{i, j}=\exp \left(-\frac{\left(\operatorname{lon}_{i}-\operatorname{lon}_{j}\right)^{2}}{L_{l o n}{ }^{2}}\right) * \exp \left(-\frac{\left(p_{i}-p_{j}\right)^{2}}{L_{p^{2}}}\right) * \exp \left(-\frac{\left(q_{i}-q_{j}\right)^{2}}{L_{q}{ }^{2}}\right)
$$

and the corresponding entry in the covariance matrix B is determined as

$$
B_{i, j}=\sqrt{B_{i, i}} \sqrt{B_{j, j}} \operatorname{Cor}_{i, j}
$$

To make the amount of nonzero B elements manageable, all correlations $\operatorname{Cor}_{i, j}$ that are less then the threshold of 0.01 , are discarded. The correlations between a particular fixed grid point and all other grid points with the same value of magnetic longitude and with the same value of $p$ are presented in

Figure 5. 

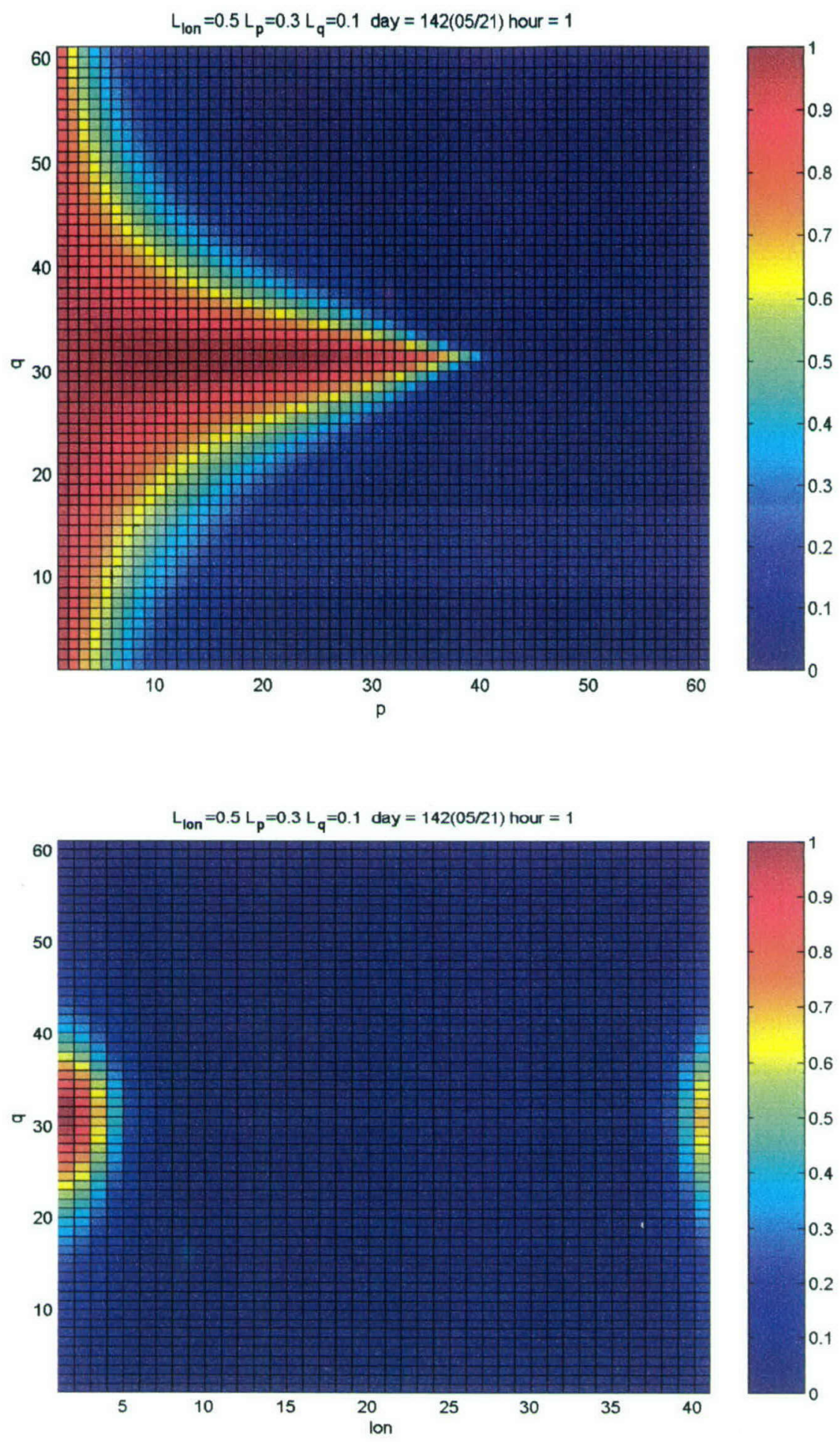

Figure 5: An example of $q-p$ and magnetic longitude - $q$ cross-sections of background error covariance between a fixed point in the model grid and all other grid points. 
The variance $B_{i, i}$ is recomputed after every Kalman Filter analysis step as follows:

$B_{a}=(I-K H) B_{b}$, where $B_{a}$ is the resulting variance, $B_{b}$ is the variance before the Kalman Filter analysis step, $\mathrm{I}$ is the unit matrix and $\mathrm{K}$ is the Kalman gain matrix, defined as $K=B H^{T}\left(H B H^{T}+O+R\right)^{-1}$, with $\mathrm{O}$ being a covariance matrix of the observational errors, and $\mathrm{R}$ being a regularizer matrix.

The time update of the covariance matrix $\mathrm{B}, B_{t+\Delta t}=F\left(B_{a}\right)$ is also performed only on the variance, with the off-diagonal elements of B rescaled accordingly afterwards, as shown above. The variance update is performed as follows:

$$
B_{t+\Delta t}^{i, i}=B_{t}^{i, i}+Q_{t+\Delta t}{ }^{i, i}=B_{t}^{i, i}+\alpha^{2} N e_{i}^{2}
$$

Here $\alpha$ is a relative model error accumulated between successive analyses and $\mathrm{Ne}_{i}$ is an electron density in the grid point $i$.

Special care needs to be taken regarding the speed and memory requirements of the implementation of the Kalman Filter equations. A set of custom data structures has been designed to store the sparse observational matrix $\mathrm{H}$, various lookup tables for the correlations between model grid points, and various intermediate matrices. In theory, it is possible to precompute all the correlations during the initialization phase. However, given that each correlation requires up to 10 bytes of storage (at least 4 bytes for the correlation value and up to 6 bytes to store information about what grid points it corresponds to), the amount of memory required to store correlations would be prohibitively large. In addition, the experiments showed that the time to retrieve the precomputed correlations can be significant, given the large size of the lookup table and the totally random manner in which correlations need to be accessed. Consequently, the correlations are mainly computed on the fly.

Such an approach could also be prohibitively expensive if performed in a straightforward fashion. Of course, not all elements of B are computed each time, but only those that have corresponding nonzero entries in the observational matrix. Physically it means that, during each assimilation step, we only need to compute the correlations between the model computational cells intersected by line of sights between receivers and satellites, and all other computational cells. In addition to the abovementioned simplification, the algorithm to compute the product of the covariance matrix B and the observational matrix $\mathrm{H}$ takes advantage of the properties of the magnetic grid and the "fractal" structure of the covariance matrix B. The algorithm also takes advantage of the fact that correlations are considered only if their value is less than a certain threshold, and uses an intelligent way to make a quick estimate of a correlation without determining its actual value. To speed up the computation, several lookup tables are created at start-up that contain, in a concise form, the information about what ranges of elements of the covariance matrix B are nonzero. This information is used in conjunction with the bounding box for a particular observation (i.e. a line of sight between a receiver and a satellite) to quickly determine the (hopefully) small subset of the correlations that actually need to be computed.

\subsubsection{Slant TEC determination from the IGS Global Positioning System data.}

The IGS (International GPS Service) Global Positioning System data is utilized to compute the receiver and satellite coordinates, and to determine the total slant TEC value along the lines of sight between receivers and satellites. The algorithm to determine the latter is similar to that described in Blewitt 
(1990). The dual-frequency GPS pseudorange data from the GPS ground stations include carrier phase and code measurements, both of which have to be utilized in determining slant TEC values. The code data, which accuracy is in the meter range, is used to determine the initial integer number of phase cycles between a satellite and a ground station. The phase data is much more accurate, with accuracy in the millimeter range, but the phase data ambiguity regarding the integer number of cycles between satellites and receivers has to be accounted for. This number is initially determined with the help of the code data, and it remains constant for as long as the receiver maintains a lock on the signal. However, the so called "cycle slips," or sharp discontinuities in the carrier phase data, that happen when a receiver fails to maintain a lock on the satellite signal, have to be accounted for. This is accomplished by maintaining a cache of approximately 40 minutes worth of previous data.

\subsubsection{Bias Determination}

Dual-frequency GPS receivers normally have large differential code biases (DCB). Small differences in the L1 and L2 hardware channels of dual-frequency receivers result in additional differential signal delays. If ignored, these biases will skew the estimated slant TEC measurements.

Quite often these biases are as large as, or even larger than, the useful signal. Typical measured TECs are in the range of $0-100 \cdot 10^{16}$ electrons per $\mathrm{m}^{2}$ (or 1-100 TEC units, TECU). Receiver biases expressed in TEC units that we have observed can easily reach $\sim \pm 50$ TECU. These biases can also change (albeit slowly) and are the effect of aging equipment or varying environmental conditions. Clearly, for the reference station data to be useful such biases need to be estimated, monitored, and removed from the measurements.

A simple way to estimate bias is to assume zero ionospheric electron content at night. The ionosphere however, can easily vary by $\sim 10$ TECU through the night and this introduces no insignificant errors in the estimated bias.

A more sophisticated way relies once again on the "thin shell" approximation. In a "nutshell," vertical TEC is simultaneously estimated at a number of ionospheric piercing points between station $i$ and satellite $j$. Then it is assumed that the TEC variations between two geographic locations are correlated. Typically, a Gaussian correlation function is used with de-correlation lengths of a few hundred kilometers specified in geographic coordinates. The sum of the unknown bias and the unknown 'true' slant TEC constitutes the measured slant TEC $[i, j]$. Unknown biases and 'true' TECs can be estimated using linear Kalman filter starting from a reasonable background guess. After enough data is accumulated and if the error covariance model and background guess are correct, uncertainties in both unknowns will decrease. A simplification of this approach assumes that nearby true TECs are the same if they are located within a certain distance. This way, if enough data are available, one can write a system of linear equations relating measurements and unknowns. If biases from one or more stations are known a priori the system can be solved and biases estimated.

The quality of bias estimation clearly depends on the validity of the thin shell approximation and, in turn, directly affects the quality of the differential ionospheric corrections, which are as well influenced by the errors of the "thin shell" approximation and errors of the interpolation.

Formally, slant TEC measured by a particular station is a sum of 'true' and unknown slant TEC and receiver and satellite biases:

$$
y_{\text {observed }}=y_{\text {true }}+b_{r}+b_{s}
$$


Depending on the local time, the magnitude of these biases is often significantly larger than the actual slant TEC. In order for the data to be relied upon these biases need to be determined and accounted for. This can be accomplished by augmenting vector $\mathbf{x}$ with both types of biases. Since the size of $\mathbf{x}$ is $\sim 10^{6}$, the number of satellite biases is $\sim 30$, and the number of IGS stations we currently use is $\sim 100$, this does not lead to any significant increase of the number of unknowns.

In principle, both satellite biases and station biases can be continuously computed using this method. At this stage in the development process we chose to use fixed broadcast satellite biases and only determine receiver DCBs. This can be justified by noting that satellite biases are perceived to be generally better known than the receiver biases and they vary at slower rate. We do plan to add continuous satellite bias calculations in the future.

Figure 6 illustrates bias adjustment results for two different stations along with DCBs determined independently by two IGS centers.
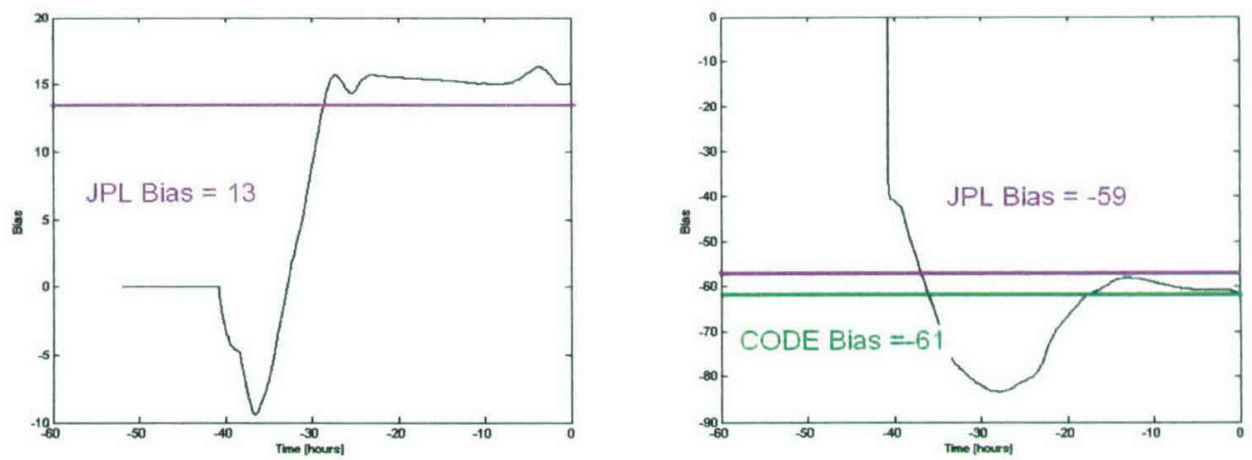

Figure 6: Examples of bias estimation obtained in the system (black line) are shown next for two different stations together with bias estimation results from other institutions when available (JPL - magenta; CODE - green).

A sample of the derived biases is shown in Table 4 for several IGS reference stations along with IGS Ionosphere Working Group computed biases. As one can see in most cases (and this is a typical for the $\sim 145$ IGS stations we used so far) the two sets of biases agree with each other to within several TEC units. Several cases with large differences are still present, however. The ultimate validation of these biases will come from systematic comparisons of the assimilation analysis produced in the system with independent data. 


\begin{tabular}{lll}
\hline \hline Station Name & Estimated biases, TEC units & $\begin{array}{l}\text { IGS Ionosphere Working Group } \\
\text { biases from JPL, TEC units }\end{array}$ \\
\hline ALBH & -23 & -28 \\
BAHR & 29 & 29 \\
CAS1 & -18 & -18 \\
DAV1 & -45 & -38 \\
FAIR & 22 & 18 \\
GUAM & 42 & 40 \\
HOFN & 10 & 13 \\
IISC & 35 & 25 \\
JPLM & -7 & -15 \\
KARR & 2 & 0.6 \\
MADR & 10 & 17 \\
NLIB & -8 & -14 \\
OHI2 & 7 & 4 \\
PERT & 19 & 18 \\
& &
\end{tabular}

It normally takes several diurnal cycles for the bias calculations to stabilize. After about 5-10 days oscillations in the biases decrease significantly. The described bias determination approach appeared to work reasonably well for a period of time. However sometimes obtained biases show large diurnal variations on the order of 10 TEC units. We attribute this to the model having a time-dependent bias as well. We are currently developing s method for simultaneously estimating model biases together with data biases in the same Kalman filter. Until this work is completed we recommend using externallyderived DCBs in operational implementation.

\subsection{Data}

There are two types of input data necessary for system's operations: static that does not change with time and dynamic that does change with time and therefore needs to be constantly updated.

\subsubsection{Static Input Data}

Static data includes the following:

- Solar EUV flux at different wavelengths that is later scaled in the model by the value of the F10.7 flux.

- Absorption cross-sections for several neutral gases.

- Photoionization cross-sections. 
- Coefficients of the World Magnetic Model 2000 (WMM 2000;

http://www.ngdc.noaa.gov/seg/WMM/DoDWMM.shtml).

- Pre-computed monthly 3-D fields of neutral composition and temperature from MSISE model. (http://nssdc.gsfc.nasa.gov/space/model/atmos/msise.html)

- Pre-computed monthly 3-D fields of neutral winds from HWM model (http://uapwww.nrl.navy.mil/models_web/hwm/hwm home.htm).

\subsubsection{Dynamic Input Data}

\subsubsection{Solar Fluxes}

One of the most important drivers for the described system is the current level of solar activity. These data (e.g., f10.7 solar flux) are obtained every 15 minutes form the NOAA Space Environment Center, http://www.sec.noaa.gov.

\subsubsection{GPS Reference Station Data}

Navigational RINEX files that contain satellite orbit information are obtained from IGS network stations archived at NASA's Crustal Dynamics Data Information System (CDDIS) with either hourly or daily delays (http://cddisa.gsfc.nasa.gov/gps_datasum.html). We currently use 1-day delayed data to simplify operations.

Daily or near-real-time reference station observational RINEX data files are also obtained from the CDDIS archive as compact RINEX and uncompressed for processing as needed

We obtain IGS Ionosphere Working Group produced satellite and receiver biases in IONEX files from ftp://cddisa.gsfc.nasa.gov/pub/gps/products/ionex. These files are only available with about a 7 day delay.

\subsubsection{Data Handling Scripts}

In order to handle updating dynamic data files we developed several redundant data-fetching scripts residing on Linux server that continuously bring in (via ftp or http) and archive the data. Data latency is monitored and recorded and operator can be alerted to possible problems.

\subsection{Data Processing and System Operations}

An overview of data processing is given in Figure 7. Data collection is independent from the model and assimilation system operation, if data is delayed for whatever reason, a warning is generated and the system operates with the most recent solar data and no data assimilation.

The model time step is 1 minute. Model time is synchronized with real-time. So occasionally the model has to stay idle to maintain synchronization. At the beginning of each time step, the most recent solar activity data is obtained and used to project current 3-D distributions of electron densities one step into the future. The system then estimates locations of all satellites and computes the slant TECs from several user-set locations on Earth to all satellites in view. Results are displayed on the web page. Additionally, a profile of electron densities corresponding to the location of an operational dynasonde at Utah State University's Bear Lake Observatory is extracted and archived for comparisons with the 
ionosonde measurements. NOAA's group accesses the profile and displays real-time comparisons on their web-page. Every hour the model 3-D fields of electron and ion concentrations, densities, and velocities are saved on disk and archived for up to five days.

When the system operates in the assimilation mode, after model calculations are completed at each time step, phase and code measurements from observational RINEX files for each receiver-satellite pair are read in and combined to obtain a vector of smoothed measured slant TEC. This vector corresponds to vector $\mathbf{y}$ in the Kalman filter equations. The resulting analyzed electron density field is partitioned between different ions in the model and these become initial conditions for the next model time step.

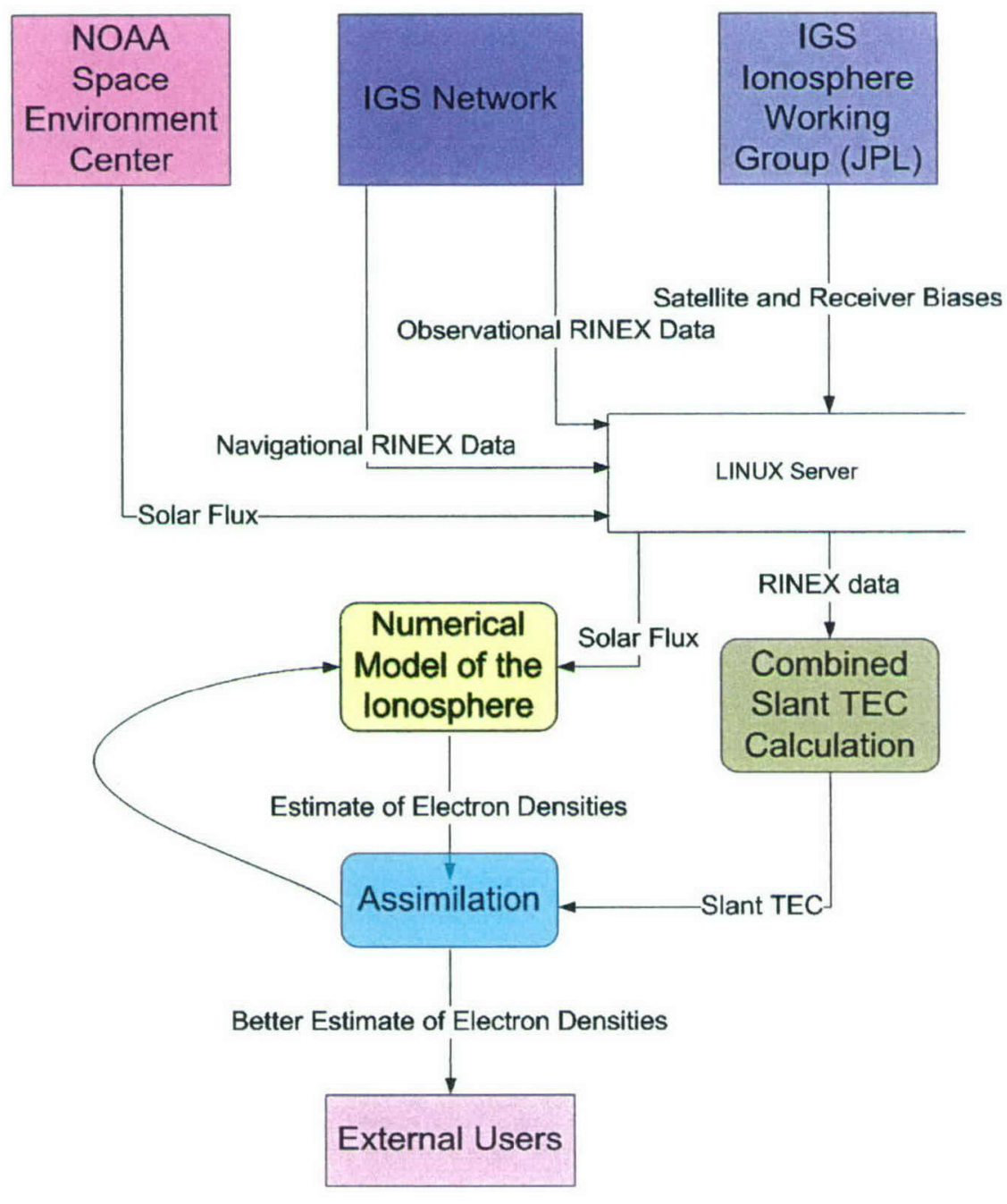

Figure 7: Data Flow in the System. 


\subsection{Ensemble Forecasting and Targeted Observations}

We explored the strategy for finding locations of "most important" observations of the electron density in the ionosphere using the ensemble prediction simulations.

The concept of adaptive observations aims to find specific geographical areas and time periods that need to be observed for obtaining the best future forecasts. The ultimate goal of adaptive (or targeted) observations is to decrease the forecast errors by placing sensors (or planning special observational modes) in regions where additional data are expected to improve the forecast. In the atmosphere and ocean studies, the strategy of adaptive observations is based on the recent progress in the understanding and analysis of predictability and sensitivity of geo-systems that were achieved through extensive applications of the ensemble based techniques. In order to identify the most sensitive regions for adaptive observations, information about the time evolution of uncertainty in the initial state, boundary conditions and the state drivers (forcing terms) should be analyzed (Farrell and Ioannou, 2004).

The Initial State (IS) uncertainty-based targeting exploits time evolution of the analysis and forecast errors to identify the most important regions for adaptive observations. Several objective techniques have been developed which promise to predict the optimal sites for additional observations. The differences between forecasts based on the routine plus adaptive observations and only routine data provide evaluation of the impact of adaptive sensors. The breeding method of Kalnay and Toth (1997), the Singular Vector (SV) techniques of Buzzia et al. (1998), and ensemble of the replicated ('perturbed') observations (Lorenz and Emanuel ,1998) have been compared and discussed in a number of previous studies. Each of these techniques has certain attractive features. For instance, the breeding method can provide information on the unstable regions of the finite amplitude perturbation without additional software development as needed for application of SV techniques. Lorenz and Emanuel (1998) concluded that the ensemble based techniques with the perturbed observations provide more consistent strategy for the adaptive targeting in the simple scalar forecast model. In the Naval Research Laboratory (NRL) the adaptive observation approach is based on singular vector and sensitivity functional algorithms obtained with the aid of the adjoint versions of global and regional operational models (Baker and Daley. 2000).

For the space weather studies the ensemble based data analysis techniques and adaptive targeting are relatively new disciplines. The direct application of the results from the numerical weather prediction studies might be erroneous because of different temporal and spatial scales of physics and chemistry. For example, the most energetic ionospheric waves present ageostrophic type of oscillations. The strong global diurnal variations create the major impact on the redistribution of the neutrals, ions, and electrons in the upper atmosphere. In comparison with the tropospheric techniques that mostly intend to target positions of unstable zones, in the ionosphere we need also to link position and timing of the fast unstable dynamics. The observed sunset equatorial scintillations present a good example of the "timespace' targeting of the unstable regions in the ionosphere. Below we describe our approach and results for adaptive observations of the electron density using the ensemble-based framework with the uncoupled ionosphere plasma model.

The uncoupled feature of the model formulation assumes the specification of the large set of the input parameters including the neutral winds and compositions, ExB drift velocities from the electrodynamics, etc. The approximate (climatological/empirical) knowledge of these input model drivers can be considered as an important source of the forecast errors of electron and ion densities.

In the data assimilation the quality of the initial conditions contribute to determining the quality of the forecast state. The traditional formulation of the adaptive observation is to study the model sensitivity to the perturbation of initial states and find the regions where the forecast errors grow quickly and linear 
perturbation of the control forecast state may be unstable. In other words, by placing the additional sensors in those unstable zones we hope to collect the most 'desirable' information that can help to improve the quality of the consecutive analysis. A breeding vectors approach is used to find locations of dominant perturbation modes using the ensemble forecast simulations. For the ionosphere plasma model the bred vectors are produced as follows: a control (base) run $\boldsymbol{B}$ is started from suitable initial conditions, then the perturbation run $\boldsymbol{P}$ is begun from the same initial state but with added random (normally distributed) perturbations of the electron density. Perturbed forecast $\boldsymbol{P}$ proceeds for 1 hour then the perturbations defined as the differences between $\boldsymbol{P}$ and $\boldsymbol{B}$ at all model grid points are renormalized using a prescribed norm. In case of the uncoupled plasma forecast model we use the initial root mean squared variance of the stochastic perturbations of the electron density. This renormalization is repeated every hour until the perturbations have formed the relatively well-defined perturbed modes. Several perturbed simulations form our ensemble prediction system. In this report we use various sizes of ensembles $(5,10$, and 20$)$ with variable strength of initial perturbations $(5 \%, 10 \%$, and $20 \%$ ). The size of the ensemble determines the local dimensions of the bred vectors $V$. In the breeding approach, renormalization of perturbations is necessary to avoid saturation of resolved unstable oscillations. It is worthy to highlight that the saturation of the noisy oscillations irrelevant to the forecast, is naturally controlled by the nonlinear breeding.

The covariance matrix $\boldsymbol{C}=\boldsymbol{V} \boldsymbol{V}^{\boldsymbol{t}}$ can be considered as a proxy for the local covariance ensemble covariance matrix. Patil et al. (2001) showed that the $\boldsymbol{C}$-matrix can be used to evaluate the 'actual' local finite-time Bred Vector (BV) dimension employing the distribution of the singular vectors of $\boldsymbol{C}$-matrix. In the numerical weather prediction the low BV-dimensions as well as a structure of the singular vectors of $\boldsymbol{C}$-matrix can indicate the most unstable subspace. As pointed out in the previous studies (e.g., Kalnay 2000) the bred vectors by construction are closely related to Lyapunov Vectors (LV) and Singular Vectors (SV) of the linear tangent model. The bred vectors share some properties of the leading LVs including the following: a) BVs are independent of the norm used to define the size of the perturbation; b) BVs are independent on the length of the rescaling time window as long as strength of the perturbation remains linear. The most significant differences between BVs and LVs can be invoked from the BV definition or construction. BV are finite-amplitude, finite-time, and because they are not globally orthogonalized, they bear the local properties in space. In the next section we present our results on the predictability and strategy of adaptive targeting for electron density observations and forecast using the described above the ensemble breeding technique.

\section{RESULTS AND DISCUSSION}

\subsection{Model Results}

In this section we give a brief overview of the model results. Since these results do not have much scientific value, only a brief description is provided to demonstrate that the model produces physically realistic output.

Figure 8 and Figure 9 show typical vertical cross-sections of densities (left), parallel velocities (middle) and temperatures for electrons and all model ions at a fixed magnetic longitude.

Typical distributions of ionospheric electron densities obtained in the model are shown in Figure 10 as a three-dimensional isosurface superimposed over the Earth. Concentrations of electrons inside this isosurface are larger than $5 \cdot 10^{12}$ electrons $/ \mathrm{m}^{2}$; electron densities outside are less than that. The radial direction has been exaggerated to show detail. 
The distribution clearly shows equatorial depletion and low-latitude enhancements that arise due to vertical transport of ions (across magnetic field lines) at the equator followed by descent along the field lines due to gravity. This is a well-known feature and the model realistically reproduces it.

Figure 11 presents latitude-longitude distribution of a typical vertical TEC in the model. Note that since high-latitude ionization processes are currently turned off, this plot does not show enhanced electron densities in the Polar regions.
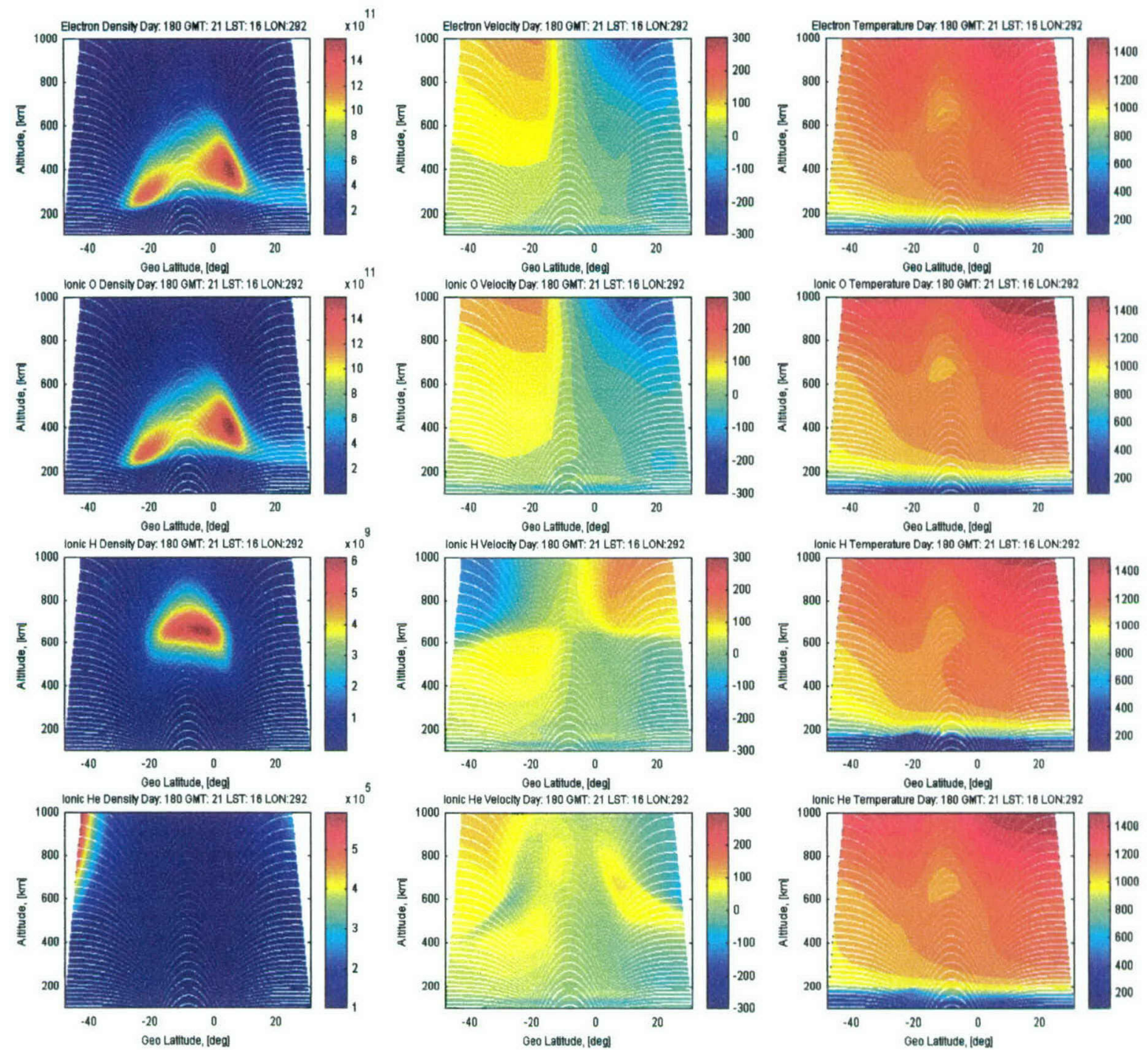

Figure 8: Examples of instantaneous model prognostic variables for electrons and major ions shown at a fixed magnetic longitude.

Real time system data are being currently downloaded to NOAA and compared operationally with the Bear Lake observatory dynasonde data. Figure 12 shows 48 hours of comparisons between modelgenerated TEC at the dynasonde location and experimental data. Detailed information on various characteristics of Bear Lake ionosonde can be accessed here:

http://www.ngdc.noaa.gov/stp/IONO/Dynasonde/. 
General features of the vertical TEC evolution are similar in both time series. Moreover, for extended periods of time the difference between the observations and the model is only about a few TEC units, which is rather encouraging. However, the model clearly does not show as rapid decay of electron densities at sunset as the ionosonde data suggest. One possible reason for this discrepancy is an absence of one or more chemical recombination mechanism in the model responsible for fast conversion of ions to neutral particles. Another possible reason is that dynasonde measurements extend only to the F-layer maxima (about $300 \mathrm{~km}$ ). A semi-empirical correction needs to be added to the derived vertical TEC to account for missing part of the profile (for, instance, via vertical extrapolation). More research is needed to properly explain this discrepancy. Also, the model nighttime TEC is generally lower than dynasonde measurements. This is likely to be due to missing nighttime ionization source. Realistic nighttime ionization will be included in the model in the near future.
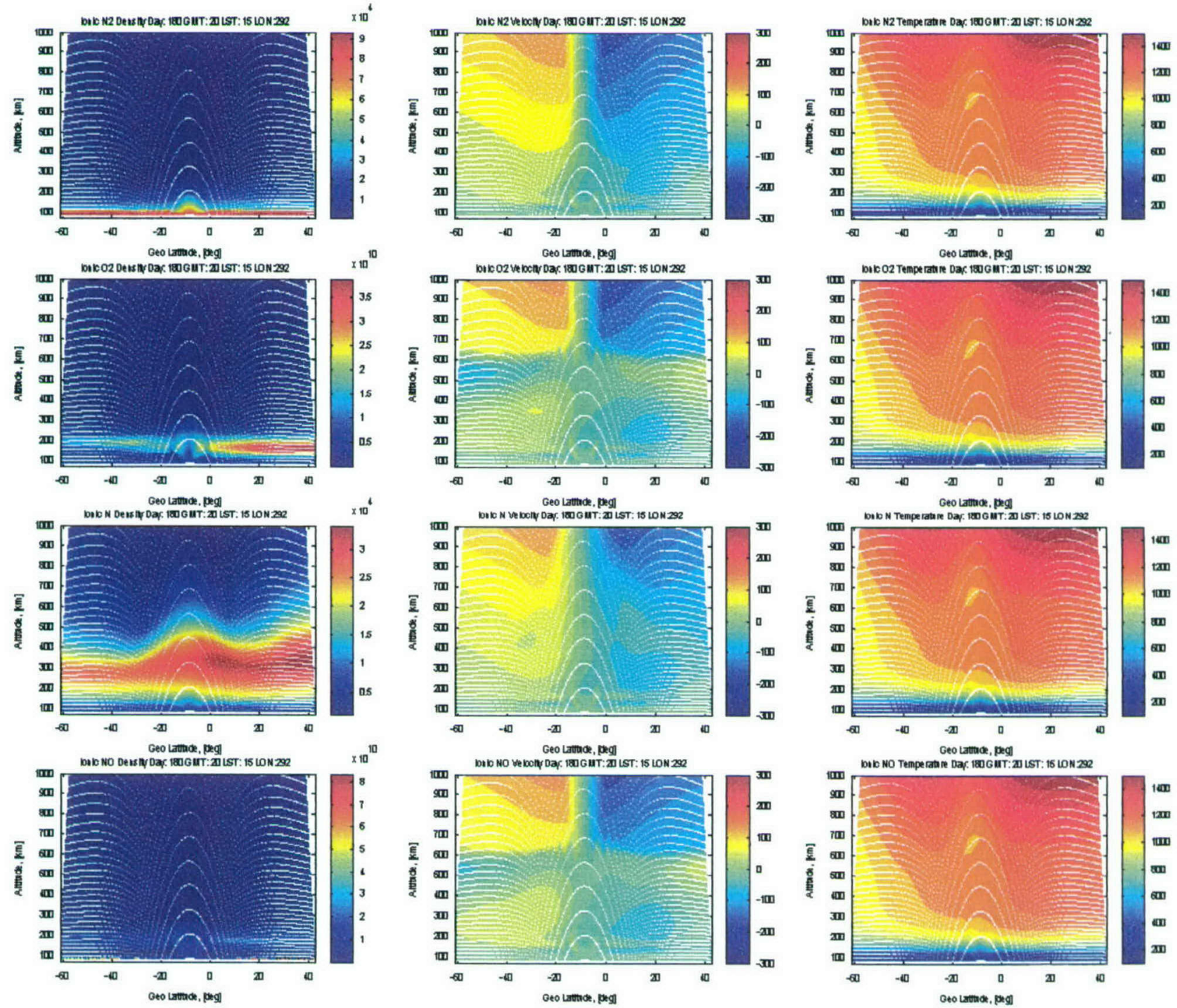

Figure 9: Examples of instantaneous model prognostic variables for minor ions shown at a fixed magnetic longitude. 

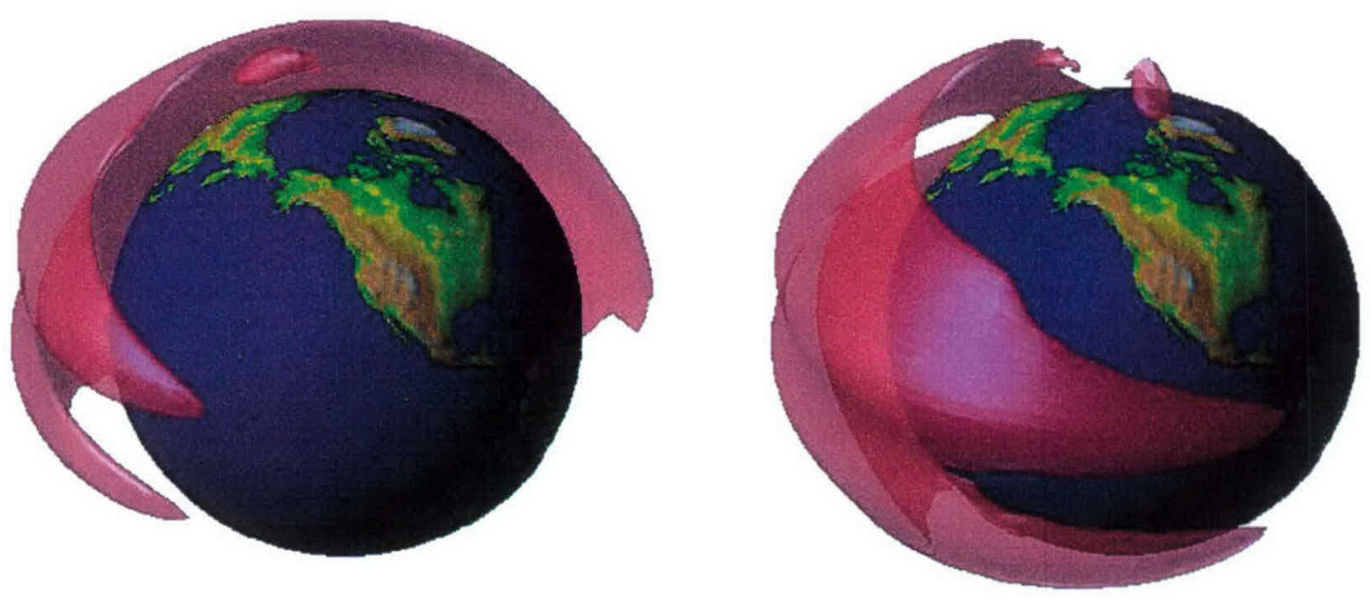

Figure 10: Examples of model-generated 3-D instantaneous iso-surfaces of electron density. The radial direction is exaggerated to show detail.
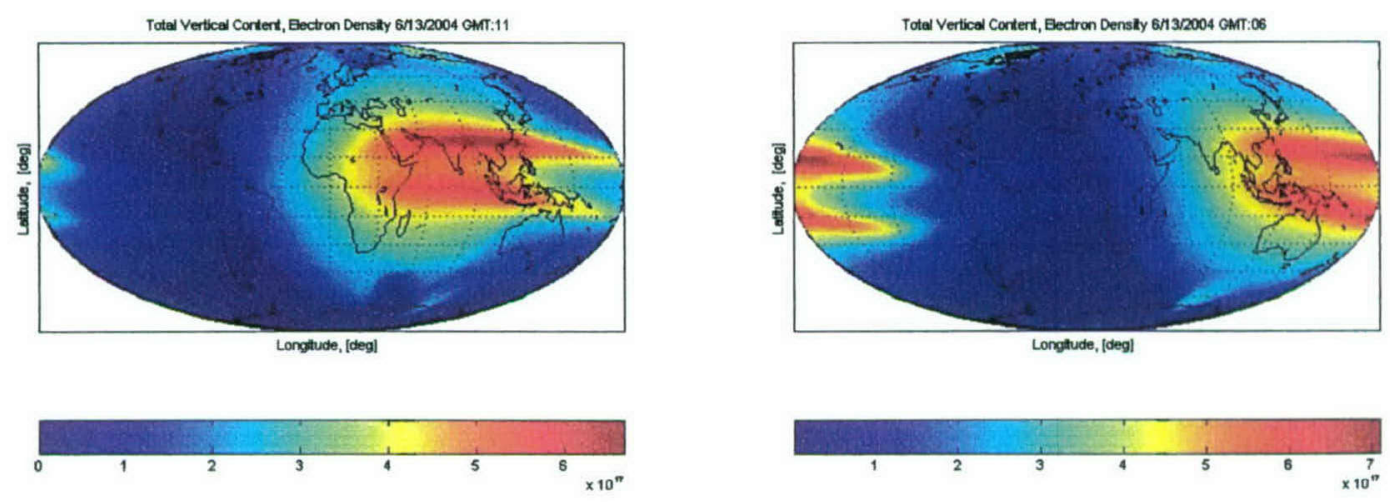

Figure 11: Examples of model-generated total vertical electron content. 


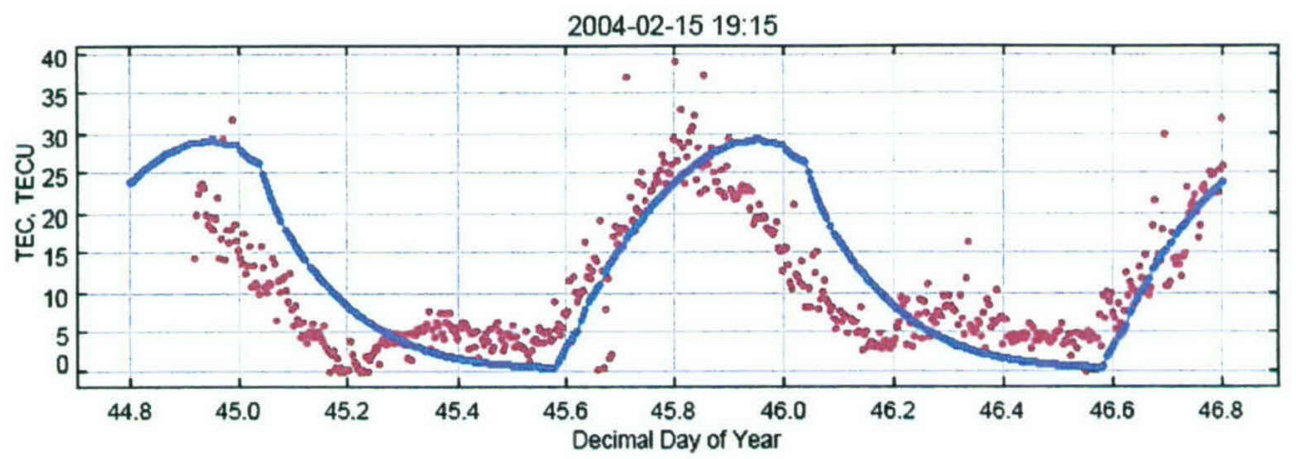

Figure 12: Time series of Bear Lake dynasonde measurements (red dots) and model simulated vertical TEC (solid blues). Courtesy of F.T.Berkey (USU's Bear Lake Observatory), J.W.Wright and N.A.Zabotin (both at CIRES/NGDC).

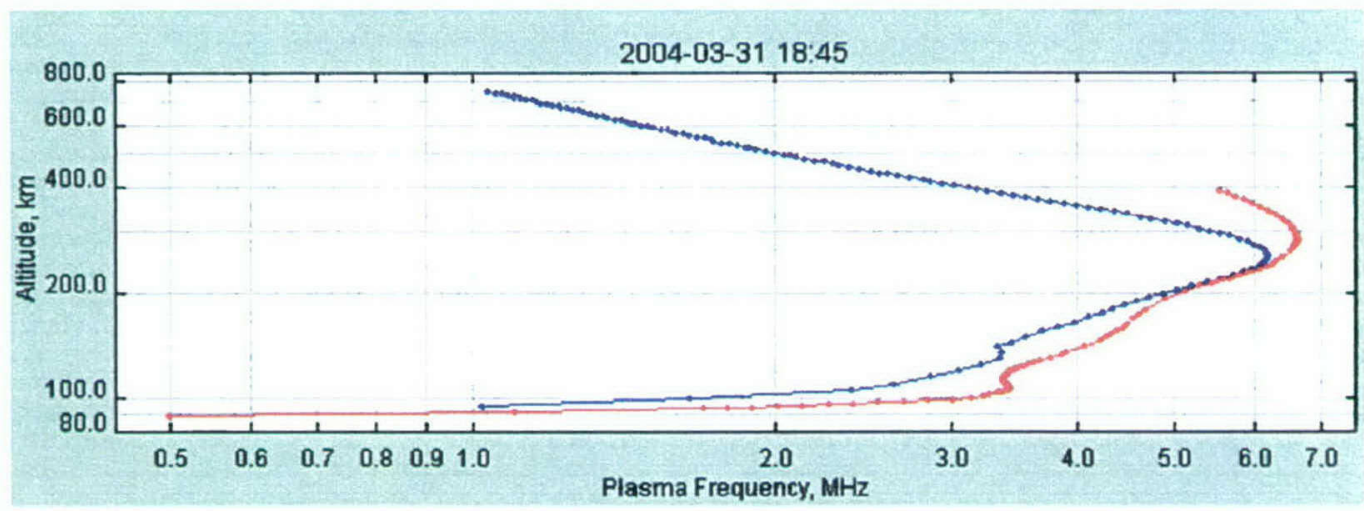

Figure 13: Electron density profile measured by Bear Lake dynasonde (red) and simulated in the model (blue). Courtesy of F.T.Berkey (USU's Bear Lake Observatory), J.W.Wright and N.A.Zabotin (both at CIRES/NGDC).

Figure 13 shows a vertical profile of electron density measured by the dynasonde and simulated by the model. Once again, while generally the plots showed the same features, some quantitative discrepancy remains.

The polar regions are characterized by a variety of distinct physical processes due to solar wind and interplanetary magnetic field penetrating the magnetosphere. Due to the extra complexity involved in modeling these processes we decided to leave some of these processes out and instead concentrate on developing the system at low and middle-latitudes. For example, auroral precipitation and heating processes are not included in the model. Nevertheless, due to importance of the high-latitude regions we included a description of the most fundamental phenomena with the goal to fill in the missing processes later.

The model grid extends to about 85 degrees for both Northern and Southern Hemispheres. Earth's magnetic field is computed in the polar regions (Figure 14) and Weimer high-latitude ExB drift model is used to obtain electric potential (Figure 15) and then ExB drift vector (Figure 16). Three components of this vector are later used for computing particle transport using a semi-Lagragian approach. An example of trajectories for three particles is shown in Figure 17. 

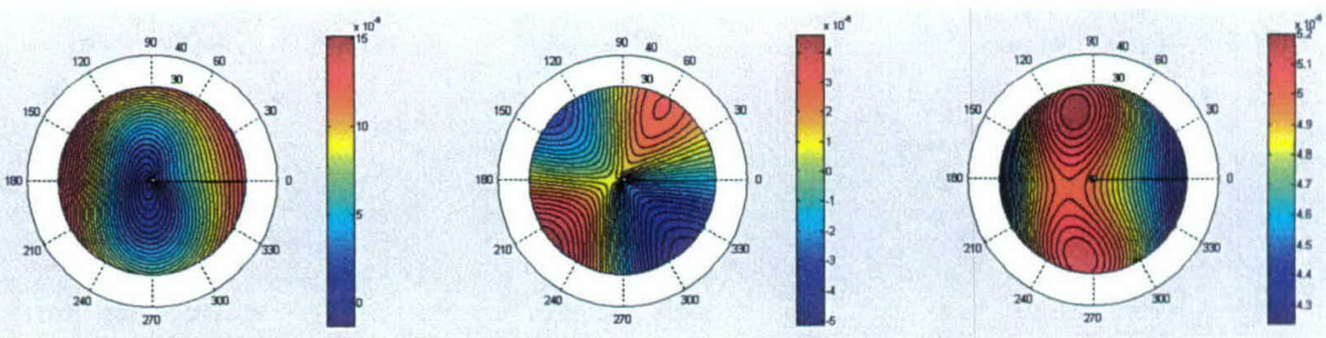

Figure 14: The $x, y$, and z-components of the Earth's magnetic field at the North Pole
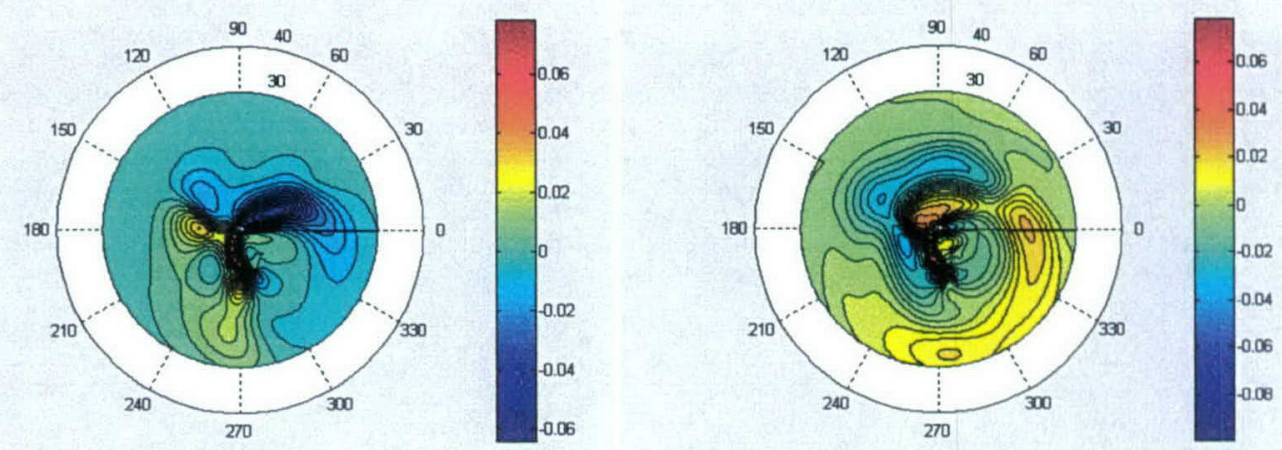

Figure 15: The $x$ - and $y$-component of the Weimer electric potential at the North Pole
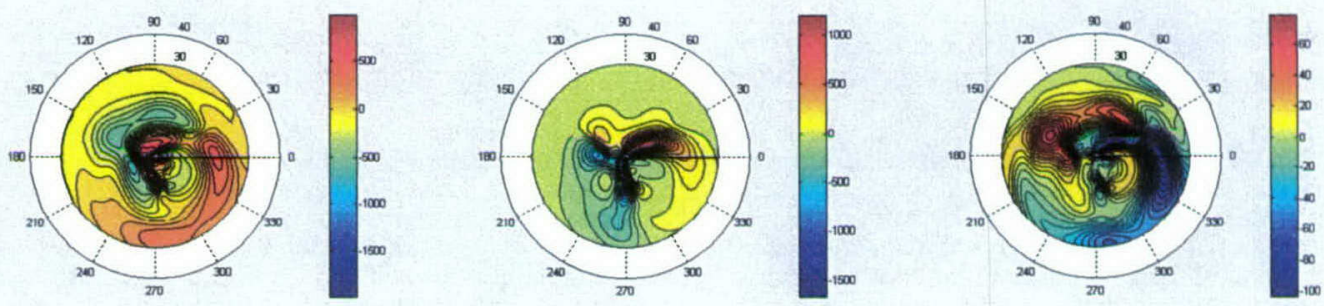

Figure 16: Calculated x-, y-, and z-component of the ExB drift velocities near the North Pole.

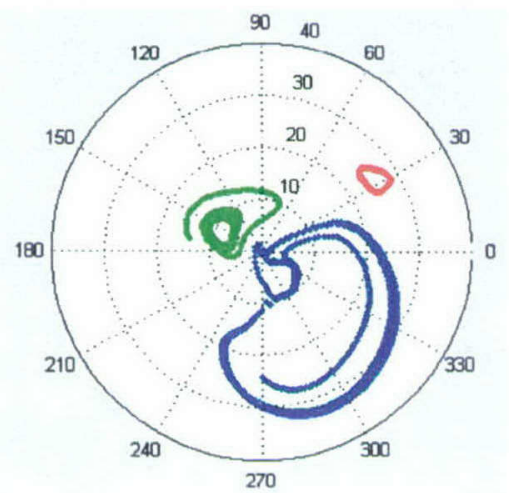

Figure 17: Trajectories of particles in the magnetic field acting under ExB drift with different starting locations near the North Pole. 


\subsection{Data Assimilation Results}

We performed a number of data assimilation runs for different days and months. Here we present several typical visualization results and later describe various statistical diagnostics and system performance indicators.

Figure 18 and Figure 19 are similar to the results of pure model integration shown in Figure 10 and Figure 11. Note however that these plots show noticeable local irregularities in response to data inputs.
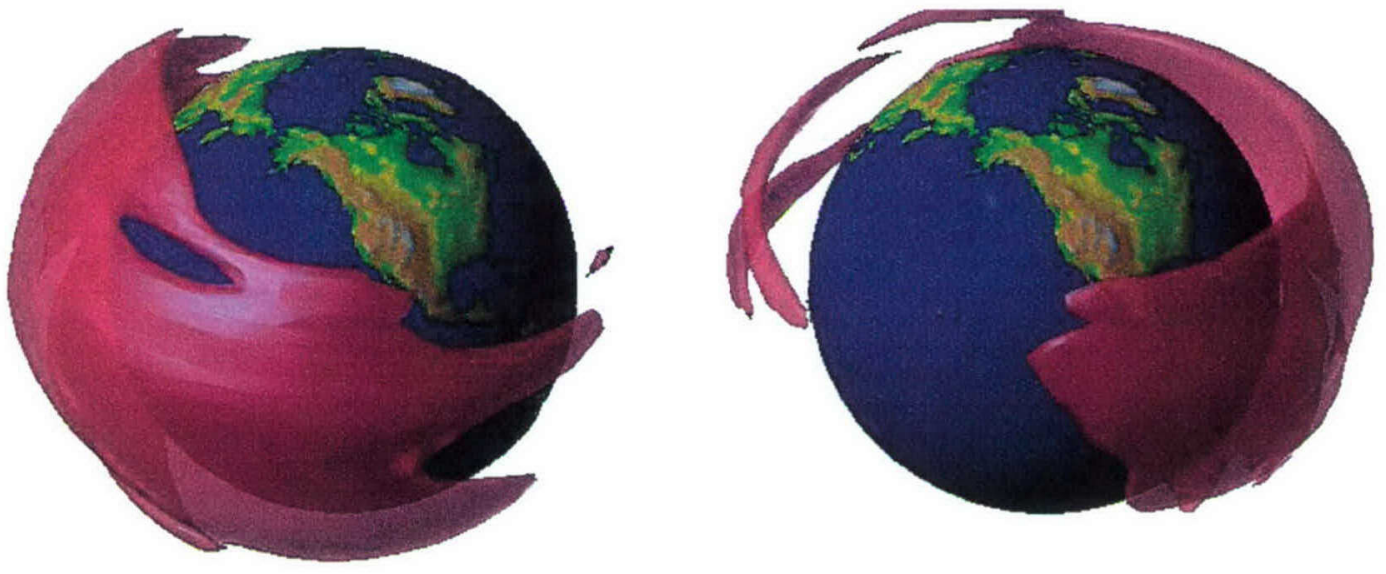

Figure 18: Examples of 3-D instantaneous iso-surfaces of electron density after assimilation. The radial direction is exaggerated to show detail.
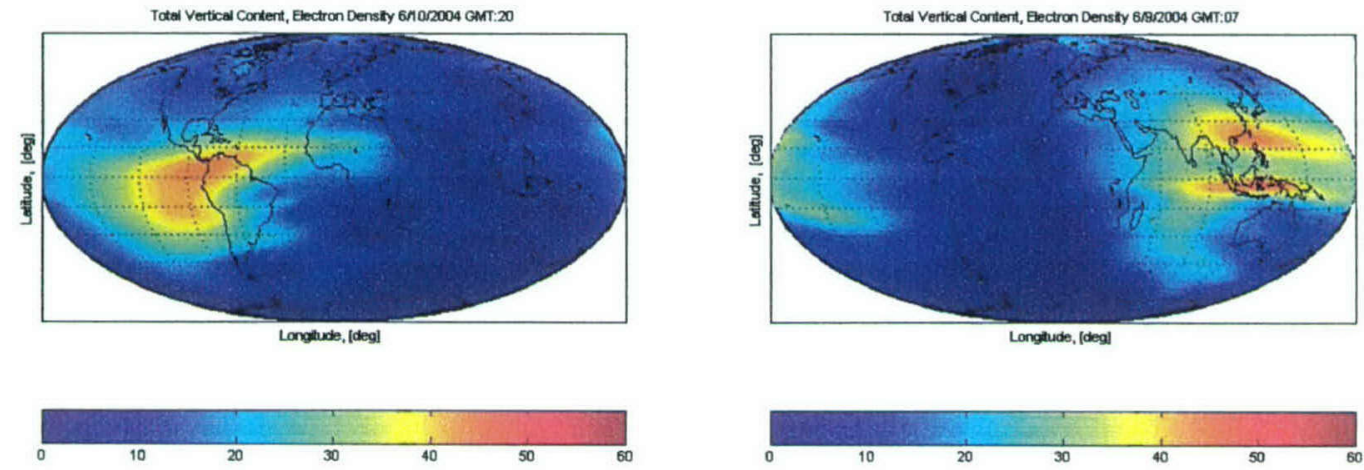

Figure 19: Examples of total vertical electron content after assimilation.

Time series of slant TECs from the assimilation and actual measurements are shown in the next figure for six different reference stations used in the assimilation. These plots are typical for all the stations we used and as one can see they demonstrate agreement to within a couple of TEC units. 

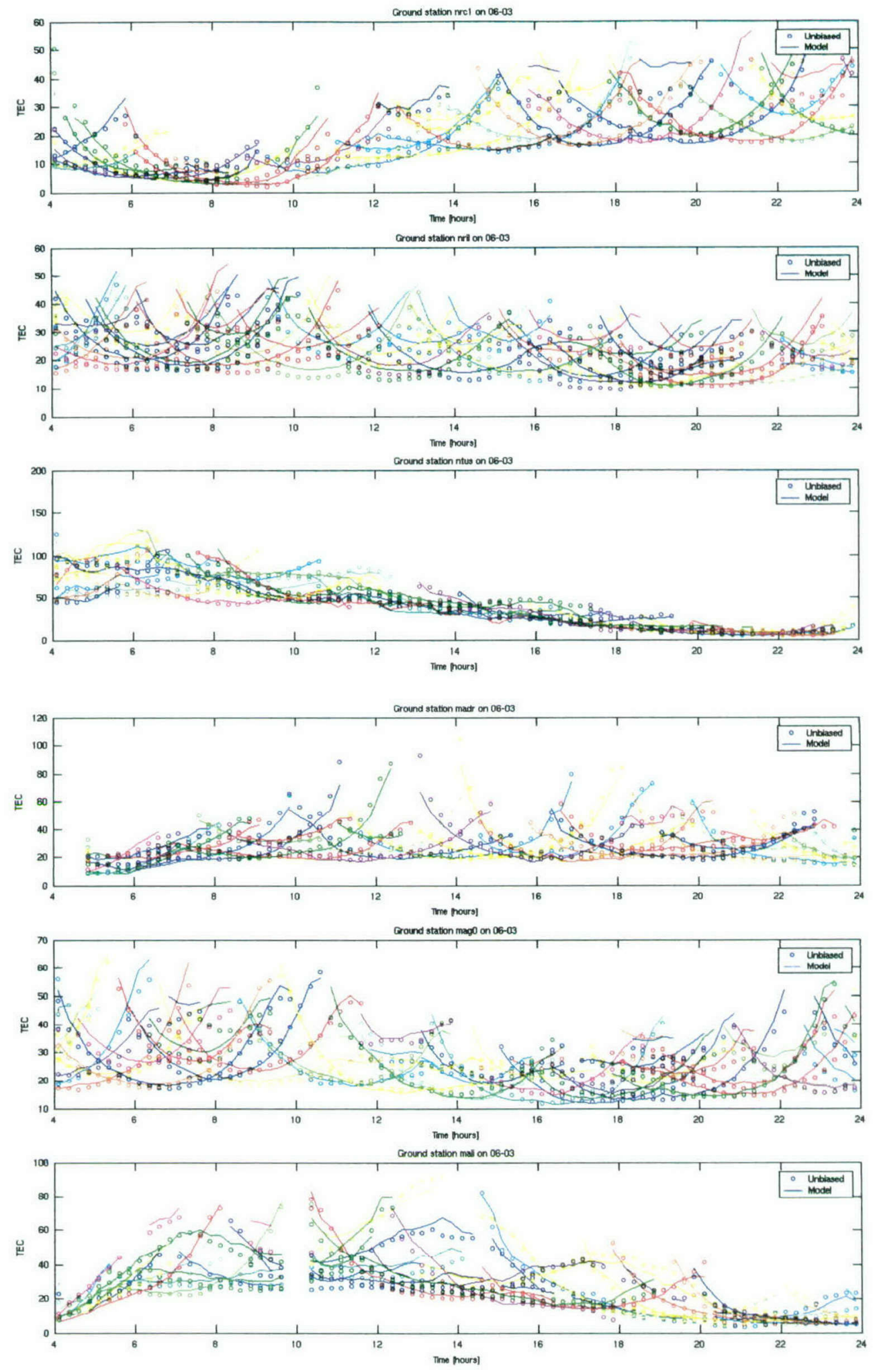

Figure 20: Typical time series of slant TEC from the assimilation for all satellites in view (different colors) for six different reference stations. 
To test the assimilation system capabilities we withhold slant TEC measurements for one or more of the IGS reference stations from assimilation. As data from other stations is being ingested into the model we sample the three-dimensional model files of electron densities along the line of sight between the control station and all satellites in view.

We only consider satellites with elevation angles higher then 15 degrees. Slant TECs computed this way are plotted for one station and several satellites in view as a function of time in Figure 21. The assimilation was turned on at the beginning of the integration and shortly afterwards the agreement between measurements and data improves. Depending on time of day, satellite elevation angle, and number of visible satellites the agreement between our nowcasts and control data proves to be as good as 1-2 TEC units.

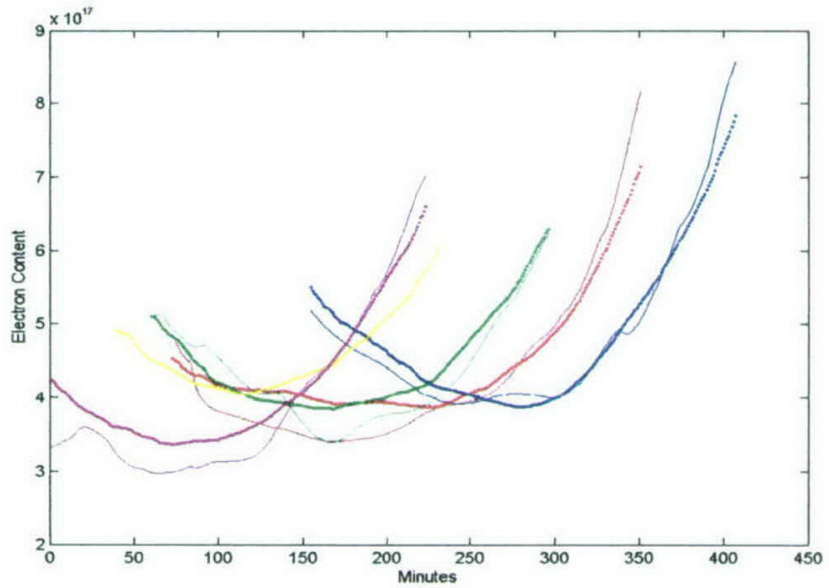

Figure 21: An example of time evolution of slant TEC from the assimilation system (solid lines) and GPS reference station measurements (dotted line) to several GPS satellites in view. Note improving agreement after assimilation is turned on at the beginning of the time series.

An important diagnostic of the correctness of the assimilation system is the so called $\chi^{2}$ (chi-squared) test. The test, described, for instance, in Khattatov et al (2000), can be thought of as a ratio between Kalman filter-estimated background (model) error and RMS residual between model and observations. As such, if tunable parameters in the assimilation scheme are chosen properly, this ratio should be on average close to 1 after initial assimilation spin-up time.

Figure 22, below, shows an example of time series. As one can see, average $\chi^{2}$ is indeed close to 1 . 


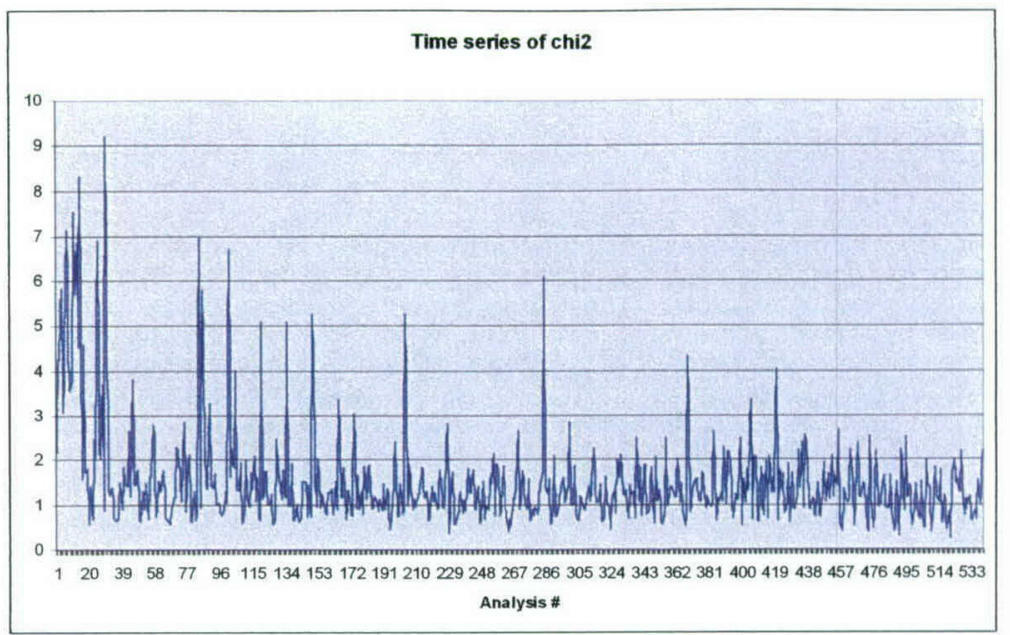

Figure 22: Time series of $0^{2}$.
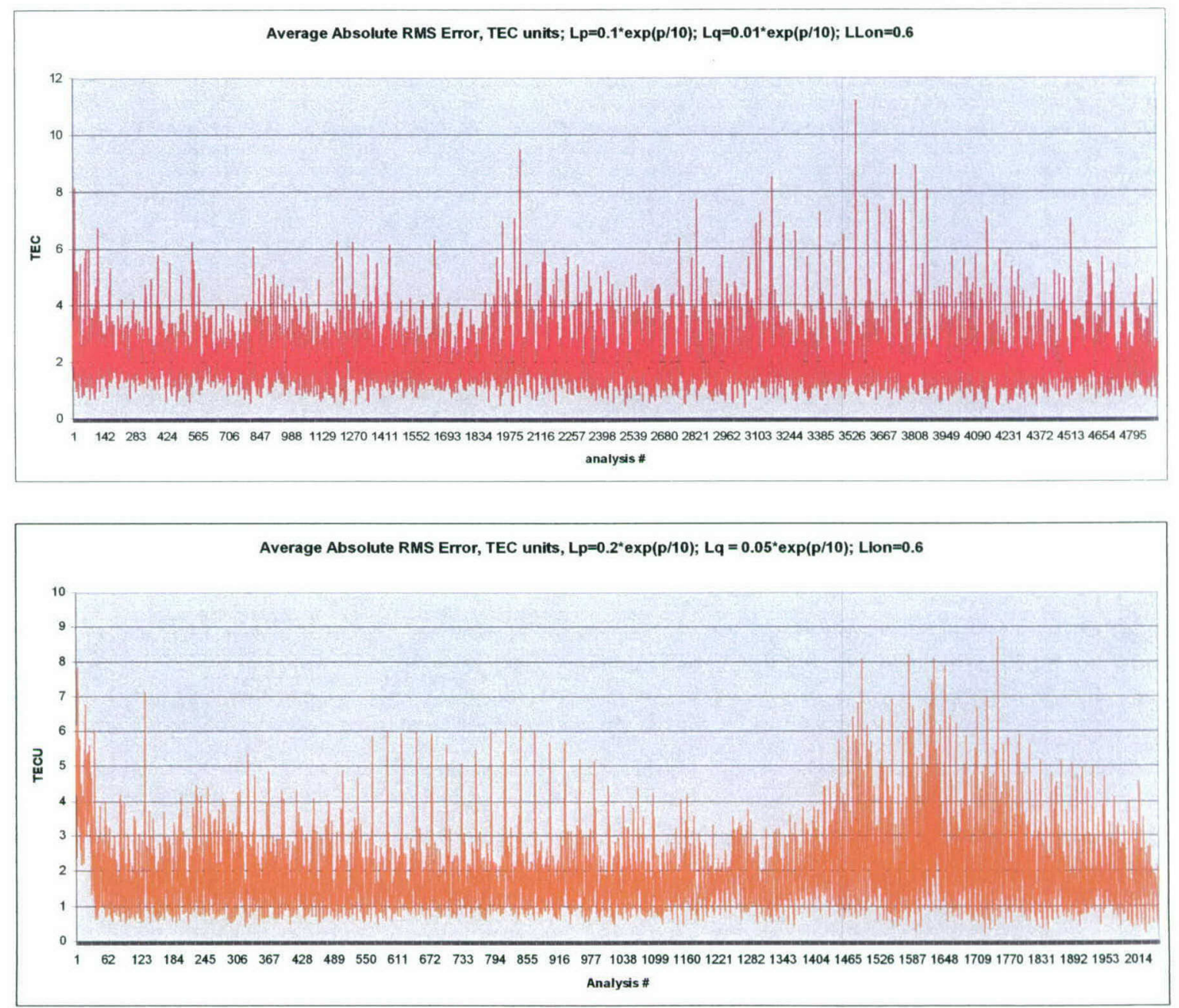

Figure 23: Average absolute root-mean squared (RMS) error for assimilation experiments with two different sets of de-correlation lengths. 
To evaluate the system we generated several measures of the assimilation system performance for all available reference stations:

- Average relative root-mean-squared differences (as a fraction) between simulated slant TEC for each receiver-satellite pair and unbiased slant TEC measurement. This diagnostic is shown in Figure 24.

- Average absolute root-mean-squared differences in TEC units between simulated slant TEC for each receiver-satellite pair and unbiased slant TEC measurement. This diagnostic is shown in

Figure 23.

- Maximum or worst absolute differences between simulated slant TEC for each receiver-satellite pair and unbiased slant TEC measurement. This diagnostic is shown in Figure 25.
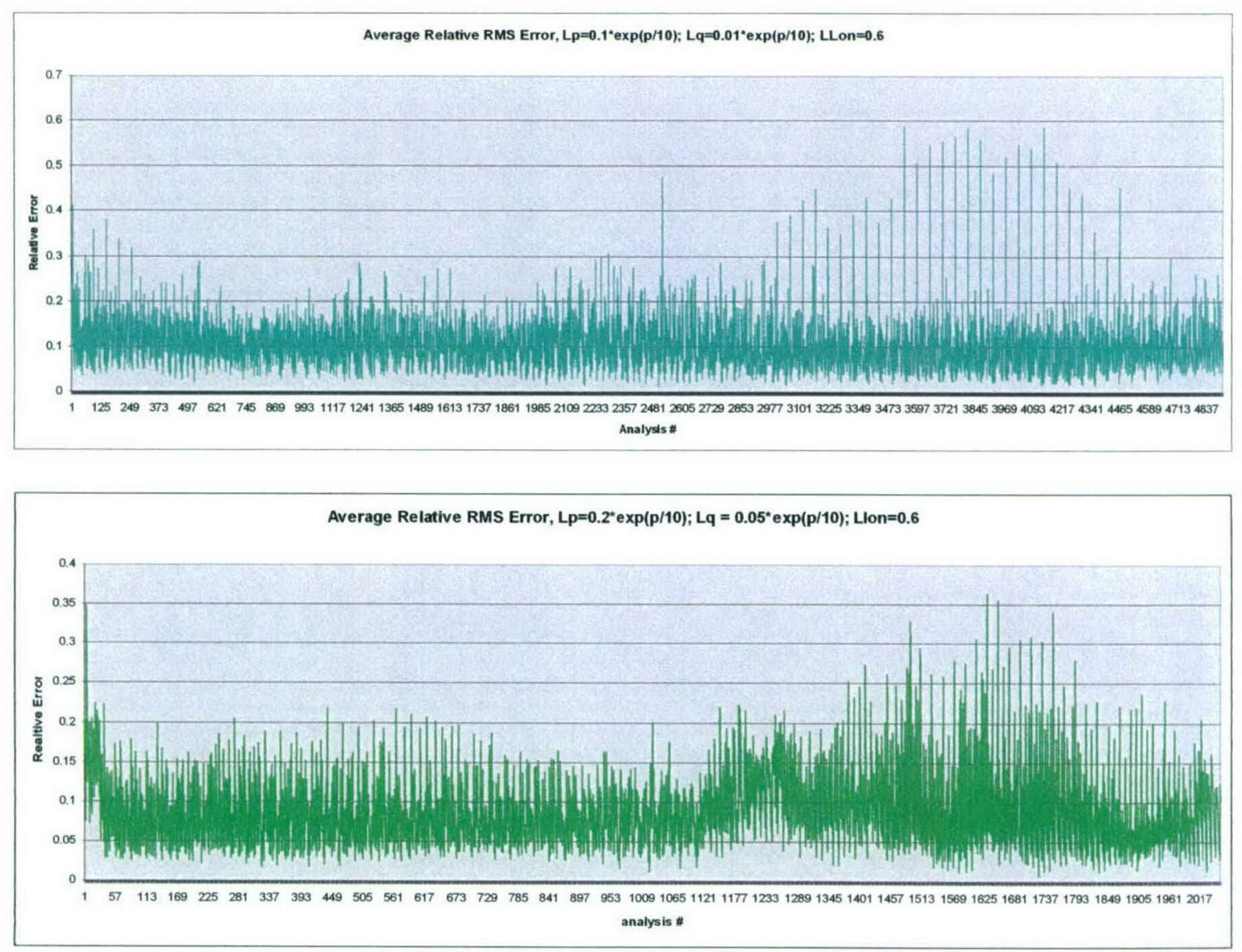

Figure 24: Average relative root-mean-squared (RMS) error for assimilation experiments with two different sets of de-correlation lengths.

In the process of finding a good set of tunable parameters for the assimilation scheme we performed assimilation experiments with several different sets of de-correlation lengths. The effect of different parameters on system performance is clearly seen in the abovementioned plots. While we plan to perform a more systematic search of the parameter space later, so far it appears that the values 
corresponding to the second set of plots are close to the optimal. They result in average RMS error of between 1 and 2 TEC units, relative RMS error of about 7\%, and maximum RMS residual of approximately 5 TEC units.

To conclude this section we note that the quality of the produced electron densities depends critically on the number of reference stations used in the assimilation. We currently use a list of 147 IGS reference stations around the world. Quite often their data comes too late or their RINEX files are corrupt. Therefore, normally not all these stations are used in the assimilation. A typical plot depicting locations of a sub-set of these 147 IGS stations that was used in the assimilation process on a particular day is shown in Figure 26.
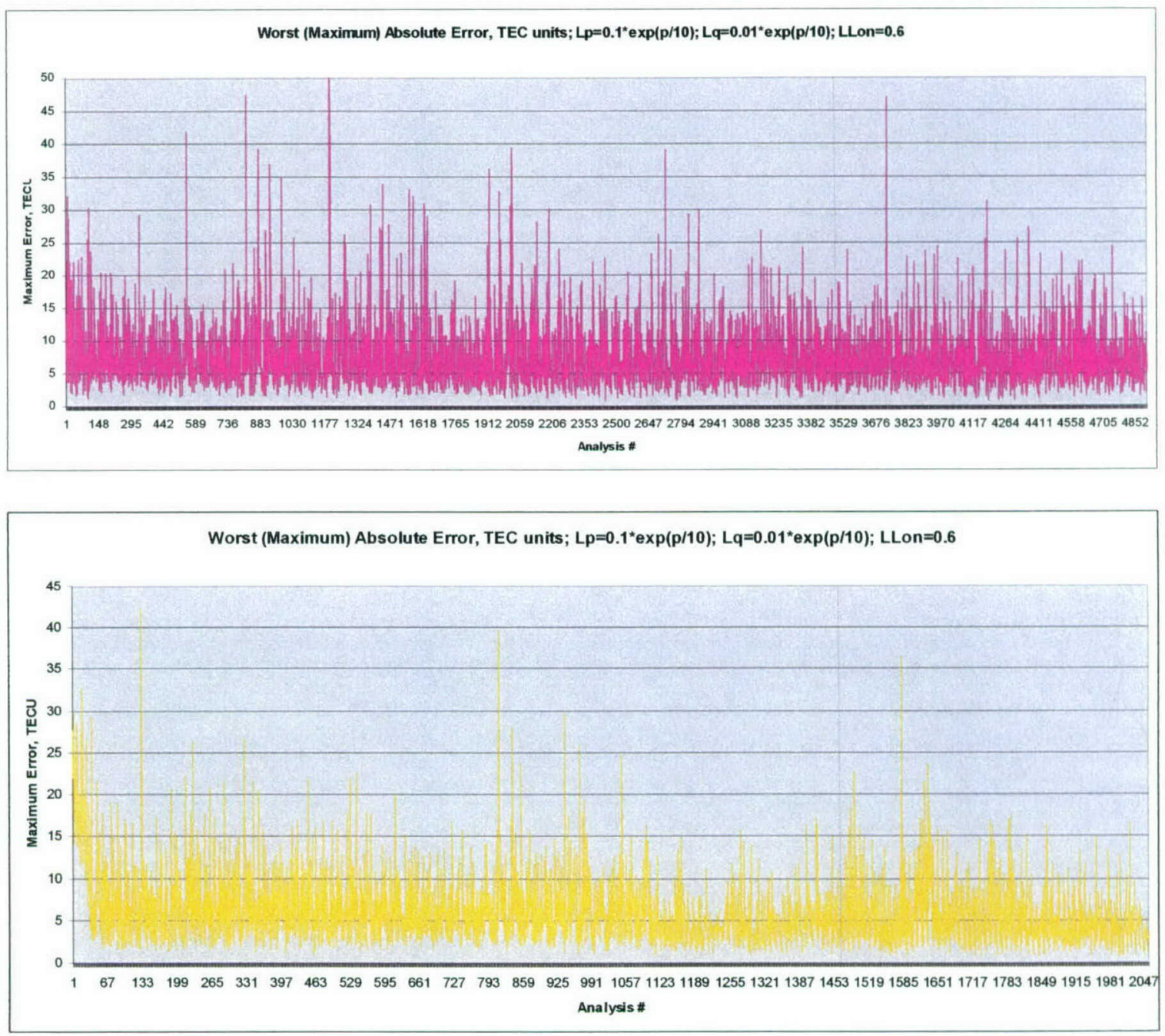

Figure 25: Maximum (worst) absolute error for assimilation experiments with two different sets of de-correlation lengths. 

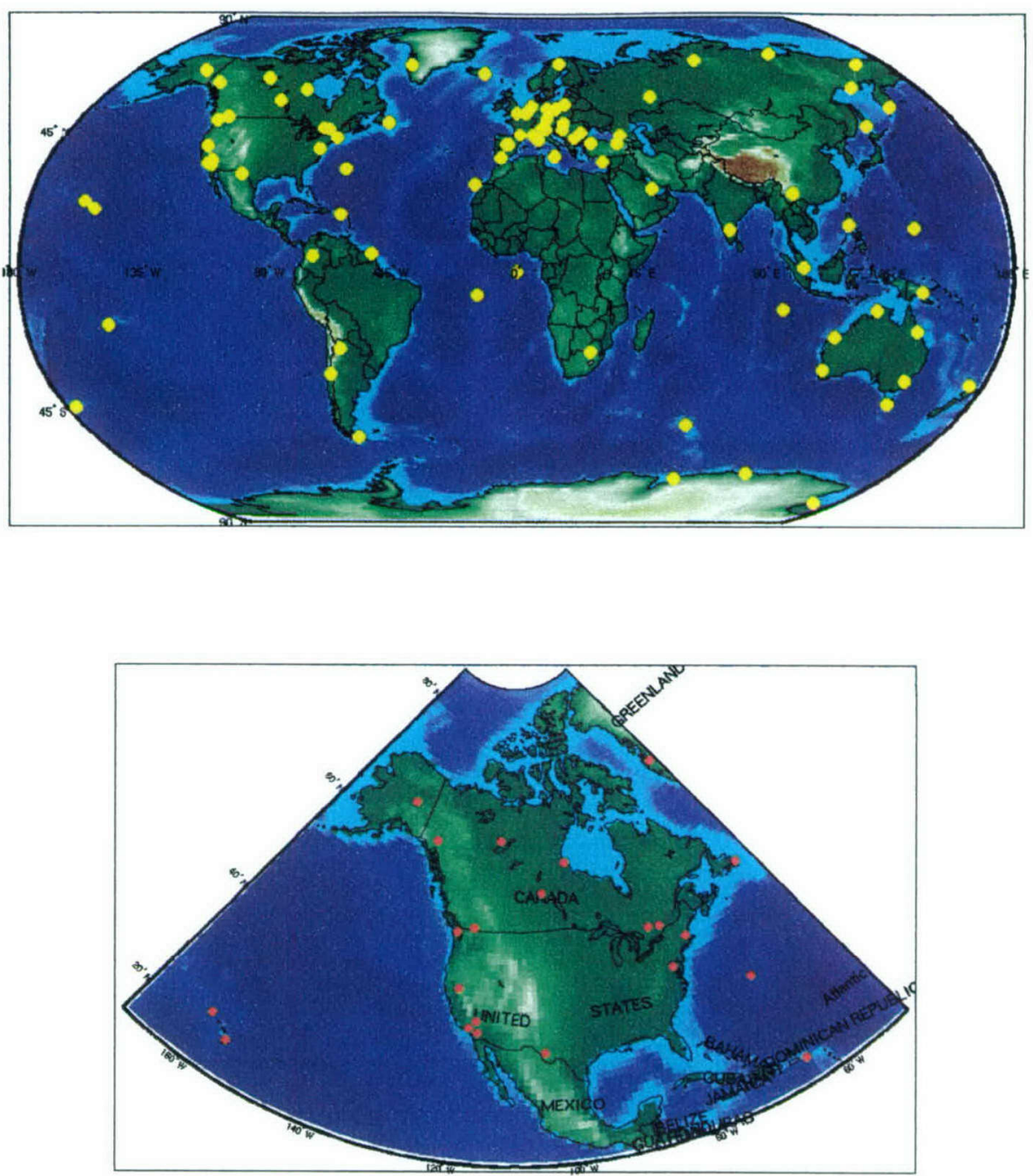

Figure 26: Locations of IGS Stations Used in the Assimilation Process.

\subsection{Ensemble Forecasting and Targeted Observations}

We carried out the ensemble forecasting with various size of ensembles (5, 10 and 20 members) and various strength of the initial perturbations $(5 \%, 10 \%$, and $20 \%)$ of electron densities. The control run corresponds to the model integration from March 4, 2004 (0 UT) to March 5, 2004 (0 UT). Every hour we applied the renormalization of the perturbations for each ensemble member. Figure 27 and Figure 28 present distributions of TEC fields after 2 and 6 hour integrations and distributions of the singular values of the bred-vector covariance matrices built with a 10-member ensemble size with various strength of initial perturbations $(20 \%, 10 \%$, and $5 \%)$. The positions of zones where the singular values are maximized do not depend on the strengths of the initial perturbations. These positions typically correspond to the sharp spatial gradients of TEC and maximum ExB-drift velocities. "Noisy" areas on these plots are due to small ensemble size dictated by computational requirements. 
TEC 2 UT

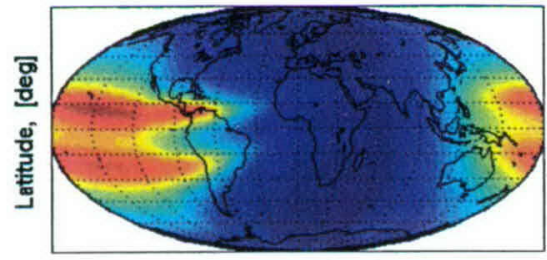

Longitude, [deg]

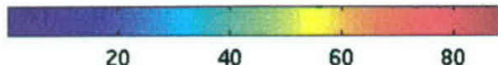

LSVD: Size $=10$, Strength $=10 \%$

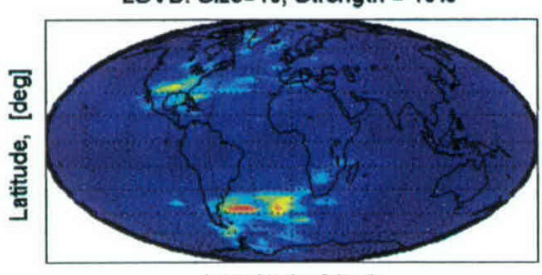

Longitude, [deg]

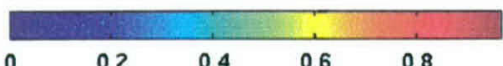

LSVD: Size $=10$, Strength $=20 \%$

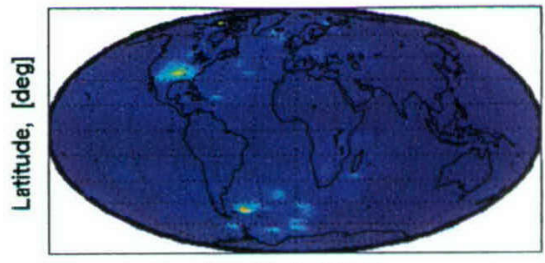

Longitude, [deg]

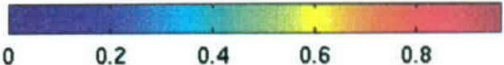

LSVD: Size $=10$, Strength $=5 \%$

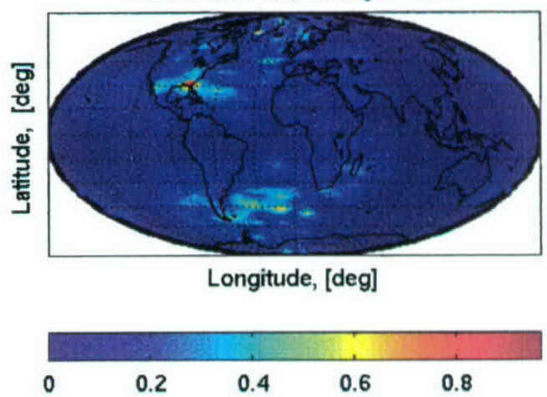

Figure 27: The distributions of TEC (a), leading singular vectors of C-matrix for $20 \%$ (b), $10 \%$ (c), and $5 \%$ (d) for initial stochastic perturbations of electron density. Results correspond to the 10 member ensemble integrations after 2 hours.
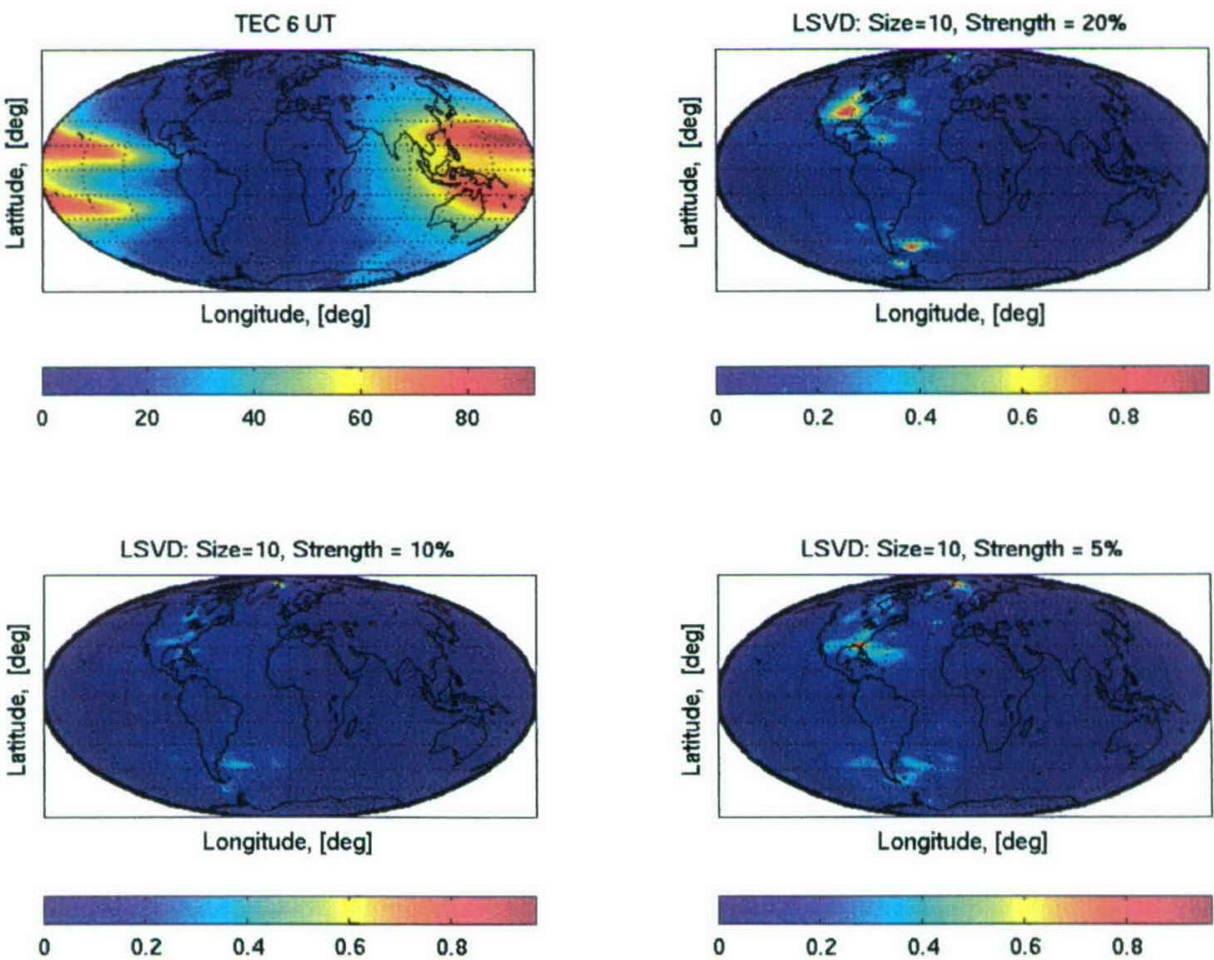

Figure 28: The distributions of TEC but after 6 hours of ensemble integrations. 
Figure 29 illustrates the longitude-altitude structure of the electron densities and singular vectors of the $\boldsymbol{C}$-matrix along the geographical equator. We can see that the shape of the singular-vector distributions does not depend on the strength of the initial stochastic perturbations. The maximum singular values are located inside the zone of the maximum vertical gradients of concentrations. The transport of the perturbations due to ExB advection plays a major role in the spreading stochastic oscillations in vertical direction. Figure 30 shows the altitude-latitude structures of the electron densities and corresponding singular vectors after 6-hour simulations. Again the spatial structure of the singular vectors depends weakly on the strength of the initial perturbations.

It is worthy to note that our results depend weakly on the ensemble size. For ensembles with 5 and 20 members (not shown) we have a similar distribution of the leading singular vectors calculated from the covariance matrices. This leads us to preliminary conclusion that the ensemble perturbation forecasting with the finite number of members (10-20) can be employed to diagnose the regions for the adaptive observations of electron density in the ionosphere.
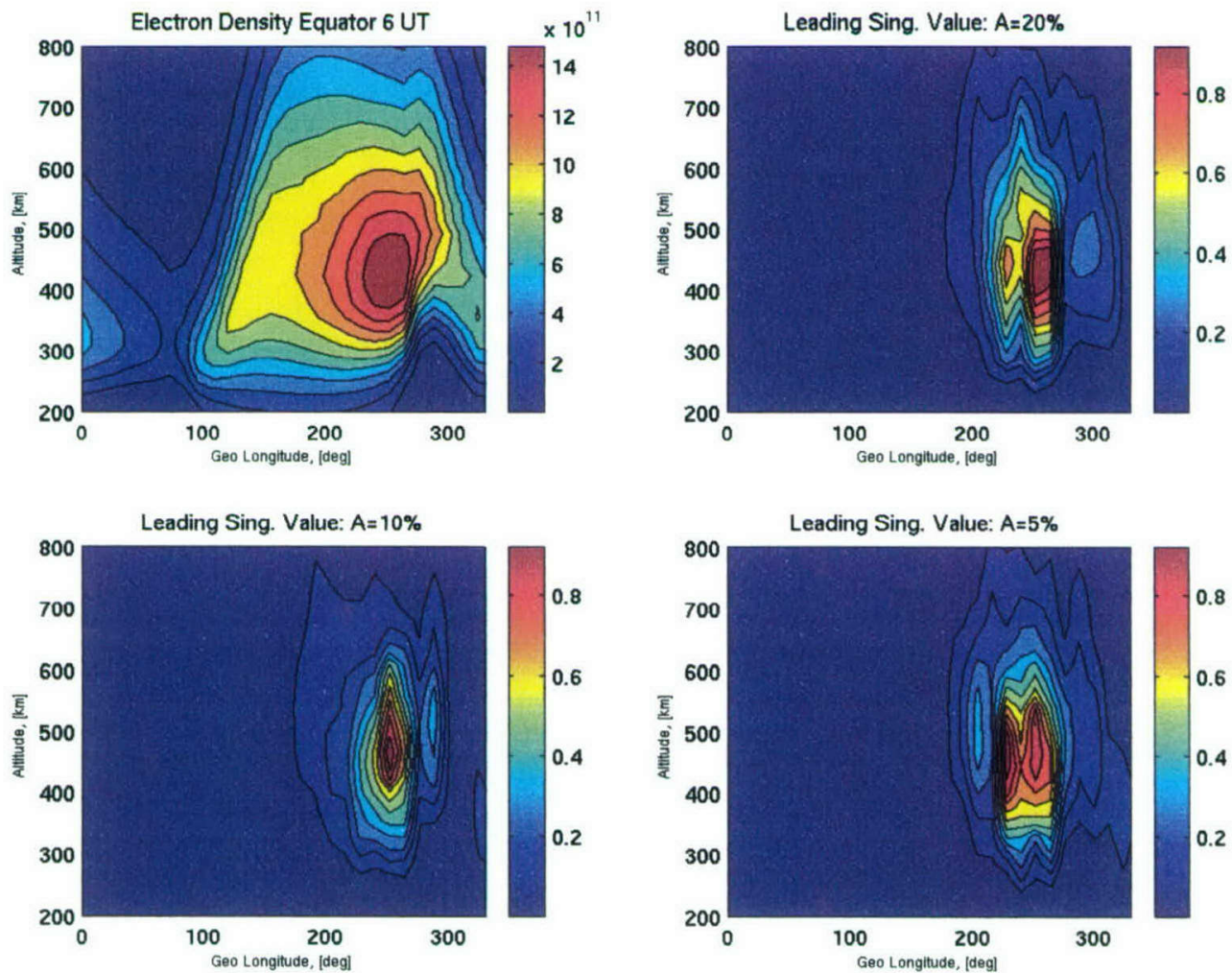

Figure 29: The longitude-altitude structure of the electron density (a), leading singular vectors of C-matrix for $20 \%$ (b), $10 \%$ (c), and $5 \%$ (d), initial stochastic perturbations of electron density. Results correspond to the 10 member ensemble integrations after 2 hours at the geographical equator.

The nonlinear plasma continuity equations that employ to forecast electron density distribution are solved along the magnetic tube coordinate system. From a practical standpoint we decided to simply start with analysis of the geographically regridded fields. This would be more convenient for determining space volumes and time windows for where and when the electron density sensors should fly. 
In the recent studies the TEC data from about 90 GPS receivers provide a main data stream for data analysis and forecast of the electron density fields in the ionosphere. Daily about 100, 000-200,000 data points (with time window every 5 minutes) can be used for global assimilation of the GPS TEC retrievals. The practical strategy for the adaptive insertion of additional GPS receivers can be invented in the course of the assimilation of existing retrievals. Depending on the data analysis scheme (variational or sequential) several strategies can be proposed to evaluate the influence function of the inserted 'adaptive' receivers on the initial forecast state. In this initial study we decided to build upon on the ensemble framework to begin study of forecast errors using the breeding approach.

Singular values of the forecast error covariance matrices based on the bred vector distribution have been used to trace out the most 'erroneous' zones for a given one hour time window. Despite on the rather complex and time consuming analysis of the ensemble simulations (their dependence on the 'starting' time of integration) the simple interpretation of results can be proposed for given forecast drivers. These drivers are the EUV solar fluxes, neutral wind, temperature, and composition, and empirical ExB magnetic tube drift model. In particular, in the equatorial F-region, a vertical ExB drift velocities in the zones of strong vertical gradients of the plasma concentrations create a vertically elongated zones of highly.uncertain forecast predictions. Near the equator, at sunset, the positions of these zones coincide with the locations of regions of the Rayleigh-Taylor instability (Kelley and Maruyama, 1992). We can expect that for the coupled ionosphere-thermosphere-electrodynamics model with the nonlinear feedbacks the proposed ensemble breeding strategy can provide a useful approach for predictability of the equatorial F-spreads as well as can help to design the special 'viewing' strategies for adaptive observations during the sunset time windows.

In the current uncoupled formulation, the forecasted plasma concentrations depend on the selected specification of the neutral thermodynamics and composition of the thermosphere as well as distribution of the electrical and magnetic fields. Specifications of these input parameters can dramatically affect the ensemble forecasting and change the positions of the most uncertain predictions. The sensitivity analysis of the ensemble forecasting to the stochastic perturbations of the major model drivers can help to evaluate changes in the position of the most uncertain zones. Although the coupled forecast ionosphere-thermosphere-electrodynamics system will be the most appropriate environment for detection of the unstable (or most erroneous) zones, the 'driver/forcing' breeding with the uncoupled ionosphere model can be used as preliminary bridge to understand the sensitivity of the plasma forecast and its error growth to stochastic variations in the external sources. We plan to continue this line of research using the ensemble perturbation framework in the assimilation of the GPS TEC retrievals.

As a final remark we should highlight a self-consistency issue between the targeting method and operational assimilation schemes. For instance, using the 4D-Var scheme it is logical to employ the adjoint-based targeting techniques and singular value algorithms described in Palmer et al. [1998]. In the sequential ensemble-based assimilation schemes the utilization of the ensemble targeting methods such as breeding or replicative observations would be the most natural strategies for adaptive observations (Lorenz and Emmanuel, 1998). At this preliminary stage of the adaptive observation strategy for the TEC retrievals in the ionosphere there is still much to investigate before to suggest some practical recommendations for the operational data assimilation schemes.

Along with the model sensitivity to the initial state, the stochastic and deterministic perturbations of the major mechanisms that control ionospheric plasma structures should be also analyzed in order to understand the relative impact of the uncertainties in the initial conditions and errors in external drivers in the TEC forecast during the day. 

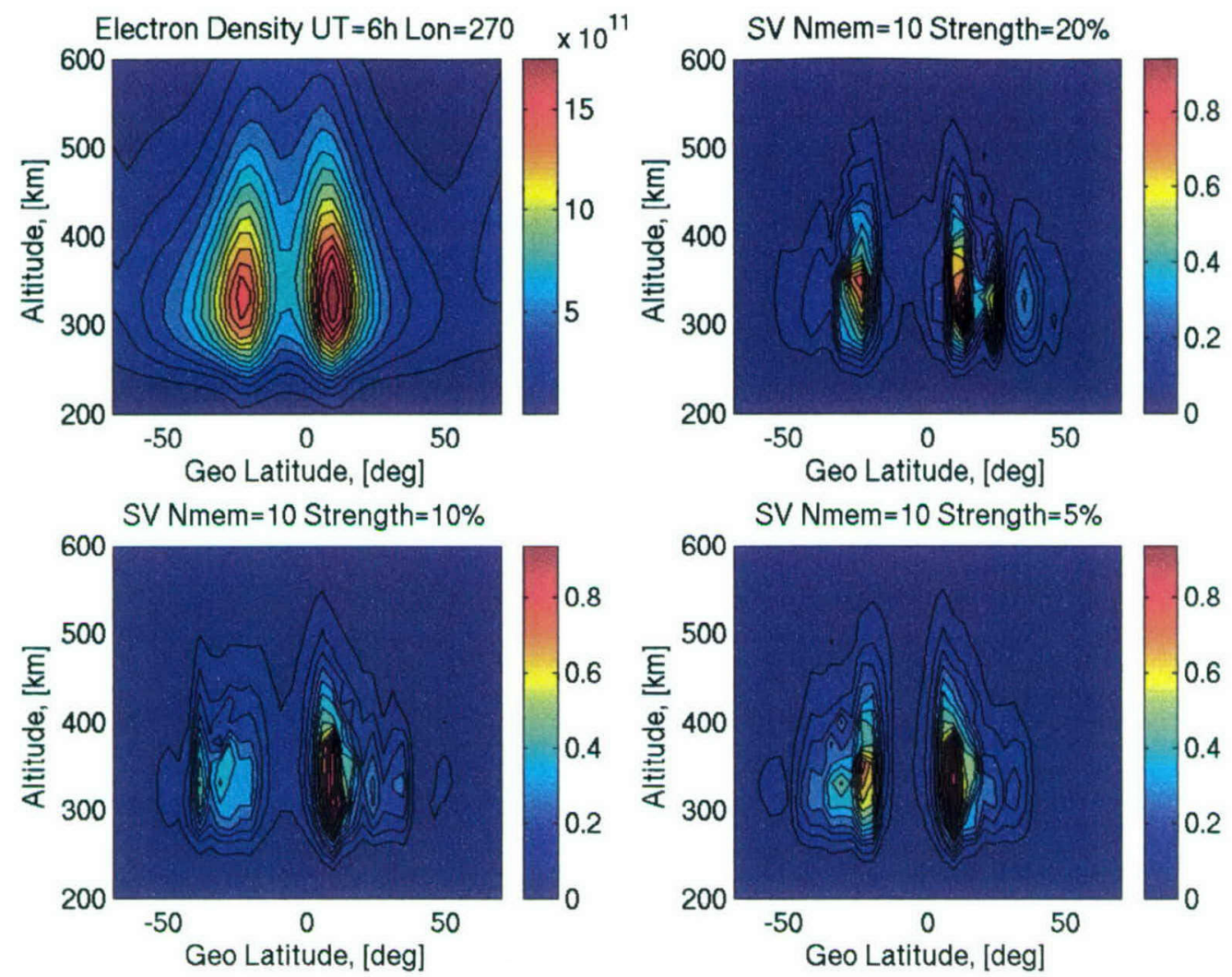

Figure 30: The latitude-altitude structure of the electron density (a), leading singular vectors of C-matrix for $20 \%$ (b), $10 \%$ (c), and 5 $\%$ (d), initial stochastic perturbations of electron density. Results correspond to the 10 member ensemble integrations after 2 hours.

\section{CONCLUSIONS}

This report presents final results of a 2-year AFRL-sponsored project whose objective was to develop advanced modeling and data assimilation capabilities for the ionosphere and upper atmosphere. In the course of this project Fusion Numerics developed a new global three-dimensional numerical model of the ionosphere, novel ionospheric data assimilation software and methodology, and an infrastructure for obtaining and managing IGS reference station data for the system.

We believe that the designed system is unique in its treatment of numerical implementation of ionospheric physics, data assimilation implementation and a modern disciplined approach to software engineering.

To the best of our knowledge, this is the only ionospheric modeling and assimilation system existing in the world that actually solves all momentum, energy and mass conservation equations and implements assimilation in model internal magnetic coordinates. Preliminary results indicate absolute average assimilation error between 1 and 2 TEC units and relative error of approximately $7 \%$. Initial control assimilation experiments performed by withholding one or more reference station data from the assimilation demonstrated accuracy within 2 TEC units. 
Fusion Numerics has received additional funding to adapt the system for assimilation of data from the future Air Force satellite mission C/NOFS (Communication and Navigation Outage Forecasting Satellite). In the course of the new development effort additional features will be developed and added into the system and system performance is expected to improve.

An operational prototype of the system has been continuously working since August 2003. The prototype uses a first-principles three-dimensional time dependent numerical ionospheric model as forward propagator and a computationally efficient large-scale Kalman filter for data assimilation and bias estimation. The system automatically acquires and archives relevant (near) real-time or delayed data from IGS network stations and the NOAA SEC center.

Differential code biases can be estimated on-line in near real time and are in approximate agreement with IGS Ionospheric Working Group supplied biases. One important advantage of our method is that these biases (and satellite biases in the future) can be made available in real time. Should unexpected changes happen at a particular reference station, (e.g., change of receiver or antenna), the system should be able to identify such change and automatically adjust for it. Under some circumstances, however, the bias determination module was known to malfunction, most likely due to systematic model biases. We are working on adding model bias estimation to the data assimilation scheme. For the time being we recommend using DCBs supplied by IGS operational centers.

We believe that these results indicate that the pursued approach is viable and has practical merit. A more extensive validation campaign is needed before such system is deemed reliable and we are in the process of setting up such a campaign with several partners.

One important capability of the system is that of not only nowcasting but also forecasting ionospheric conditions. While in principle a forecasting system is easy to set up, as it is not that different from the current configuration, rapidly changing ionospheric conditions impose high computational requirements. In conventional weather prediction forecasts are usually generated every several hours since, say, humidity or temperatures do not change significantly in between. Ionospheric electron densities change rather abruptly at sunrise and sunset. Quite often updates of 1-10 minutes might be required. Considering that each update requires a separate forecast, this can result in significant computational requirements. The problem of delayed data makes it necessary to perform forecasts fast enough to cover several model hours in a fraction of wall-clock time. On the other hand, these computations are inherently parallel and can certainly be performed if enough processing nodes are available. 


\section{REFERENCES}

Baker, N. L., and, R. Daley (2000): Observation and background adjoint sensitivity in the adaptive observation-targeting problem. Quart. J. Roy. Meteor. Soc., 126, 1431-1454.

Bailey and Balan, A low-latitude ionosphere-plasmosphere model, in STEP: Handbook of Ionospheric Models, STEP Report, editor R.W. Schunk, 1996.

Buzzia et al. (1998): The singular vector structure of the atmospheric general circulation. J. Atmos. Sci., 52, $1434-1456$

Blewitt, G. (1990): An Automatic editing algorithm for GPS data. , Geophys, Res Letters, vol. 17, No. 3, pp 199-20

Farrell, B. F., and P. J. Ioannou, (2004): Distributed forcing of forecast and assimilation error systems, paper submitted to the J. Atmos. Sci., 2004

Fejer, B. G., and L. Scherliess (1995): Time dependent response of equatorial ionospheric electric fields to magnetospheric disturbances, Geophys. Res. Lett., 22, 851-854.

Fuller-Rowell T.J., D. Rees, S. Quegan, R.J. Moffett, M.V. Codrescu, and G.H. Millward (1996): A coupled thermosphere ionosphere model (CTIM). Handbook of Ionospheric Models, STEP Report, editor R.W. Schunk.

Haji, George A. and Wilson, B. D. and Wang, C. and Pi, X. and Rosen, I. G. (2002): Ionospheric Data Assimilation of Ground GPS TEC by Use of the Kalman Filter. Submitted to Radio Science.

Huba et al. (2000): SAMI2 is another model of the ionosphere: a new low-latitude ionosphere model, $J$. Geophys. Res., 23,035.

Kalnay E. and Z. Toth (1997): Ensemble forecasting at NCEP and the breeding method Mon. Wea. Rev., 125, p. 3297-3319.

Kalnay E. (2003): Atmospheric modeling, data assimilation, and predictability, 341 pp., Campridge, University Press.

Kelley, M. C., and T. Maruyama (1992): A diagnostic model for equatorial spread F, J. Geophys, Res, 97, $1271-1277$.

Khattatov, B.V., J.-F. Lamarque, L. V. Lyjak, R. Menard, P. F. Levelt, X. X. Tie, J. C. Gille, G. P. Brasseur, (2000): Assimilation of satellite observations of long-lived chemical species in global chemistry-transport models, J. Geophys. Res., 105, 29135.

Khattatov et al. (2004): Ionospheric Corrections from a Prototype Operational Assimilation and Forecast System, Proceedings of IEEE Position, Location, and Navigation Symposium, (PLANS), Monterrey, CA, April 26-29.

Lorenz, E. N., and K. A. Emanuel (1998): Optimal sites for supplementary observations: Simulations with a small model, J. Atmos. Sci., 55, 399-414.

Millward et al. (1996): A coupled thermosphere-ionosphere-plasmosphere model (CTIP), in STEP: Handbook of Ionospheric Models, STEP Report, editor R.W. Schunk .

Patil D. J. et al. (2001): Local low dimensionality of atmospheric dynamics, Phys. Rev. Lett.., 86, 58785881 .

Schunk and Nagy (2000): The Ionospheres, Cambridge University Press, 2000.

Vannitsem, S., and C. Nicolis, (1997): Lyapunov vectors and error growth patterns in a T21L3 quasigeostrophic model, J. Atmos. Sci., 54, 347-361.

Weimer, D. R. (1996): A flexible, IMF dependent model of high-latitude electric potentials having "space weather" applications, Geophys. Res. Lett., 23, 2549. 
